\title{
Gradhiva
}

GRADHI

Revue d'anthropologie et d'histoire des arts

32 | 2021

Livres sorciers

\section{La parole perdue. Sur les sciences occultes dans la seconde moitié du XIX ${ }^{\mathrm{e}}$ siècle}

The Lost Word

\section{Giordana Charuty}

\section{(2) OpenEdition}

Journals

Édition électronique

URL : https://journals.openedition.org/gradhiva/5318

DOI : $10.4000 /$ gradhiva.5318

ISSN : 1760-849X

\section{Éditeur}

Musée du quai Branly Jacques Chirac

\section{Édition imprimée}

Date de publication : 24 mars 2021

Pagination : 24-47

ISBN : 978-2-35744-132-3

ISSN : 0764-8928

Référence électronique

Giordana Charuty, "La parole perdue. Sur les sciences occultes dans la seconde moitié du xixe siècle », Gradhiva [En ligne], 32 | 2021, mis en ligne le 02 avril 2021, consulté le 25 mai 2021. URL : http://journals.openedition.org/gradhiva/5318; DOI : https://doi.org/10.4000/gradhiva.5318 
Dossier

La parole perdue

Sur les sciences

occultes dans

la seconde moitié

du XIX siècle

Mots clefs:

Stanislas de Guaita,
Joséphin Péladan, Eliphas Lévi,

hiéroglyphes, primitivisme,

sciences occultes

Giordana
Charuty 
Dans la seconde moitié du XIX siècle, les adeptes d'une «Haute Magie» se réclament de traditions intellectuelles et religieuses oubliées, disqualifiées et redécouvertes à travers une pratique et un savoir de bibliophile. Cet article examine la constellation de figures autour du «mage» Stanislas de Guaita (1861-1897) qui revendique cette part maudite de la raison à partir de l'édification d'une très précieuse collection de textes renaissants et modernes. Tous partagent un même souci de réappropriation du passé de tous les livres, manifestent la même passion pour la diversité des signes et des codes graphiques, et se posent en fondateurs de multiples ordres et sociétés secrètes. Rétablir la dimension concrète de formes de vie placées sous le signe distinctif de la «Haute Magie» conduit à explorer la recomposition de rôles rituels en attente d'interprétation et à interroger les registres expressifs ainsi que les déplacements dans la littérature d'une forme paradoxale de primitivisme religieux.

1. Du français gramoire, altération de grammaire, qui désigne au xII siècle la grammaire latine inintelligible pour le vulgaire. Présents en Europe à partir des $\mathrm{XII}^{\mathrm{e}}-\mathrm{xIII}{ }^{\mathrm{e}}$ siècles, imprimés dès le $\mathrm{xVI}$ siècle, ils entrent, au siècle suivant, dans la «Bibliothèque bleue», une formule éditoriale qui réunit des textes d'astrologie, des chansons de geste, des contes et des secrets de «magie noire». Malgré de multiples

interdictions, cette littérature atteint les villages dans les ballots des colporteurs, puis des imprimeurs spécialisés les diffusent par voie postale, à partir des années 1880 .
J'aurais à savoir deux niveaux dans la parole. Je pourrais, sous celui des articulations conceptuelles, puiser dans un plus profond, l'être même, avec ces vocables d'une langue au sein de la langue qui sont ce dont les religions se souviennent quand elles parlent d'un verbe. (Yves Bonnefoy, L'Écharpe rouge)

Dans les années 1970, historiens et ethnologues de l'Europe rencontraient le livre de maǵie à la croisée de pratiques d'inégales ampleur et légitimité : les appropriations populaires d'une culture de l'imprimé, l'exercice d'un registre singulier de violence symbolique, l'agression sorcellaire. Là même où le plus diffusé de ces livres, L'Albert, modelait l'imaǵinaire des pouvoirs magiques, ce sont d'abord les effets d'une censure religieuse, réitérée depuis plusieurs siècles, que l'on enregistrait. "Ici, on l'a jamais vu, ce livre», assurait-on avant de décrire l'emprise, proche de la folie, subie par d'imprudents lecteurs aux prises avec un objet immaîtrisable qui permettait, en revanche, à un usager qualifié de réaliser les désirs de vie et les vœux de mort de ses consultants. L'attribut mythique du maǵicien ne serait-il pas la mémoire sociale de l'irruption de l'imprimé et une variation sur la lecture comme maǵie sociale (Fabre 1985)?

Nous retrouvons ces livres, que la mémoire paysanne assurait n'avoir jamais vus, au sein d'une production libraire - les «sciences occultes» - qui a nourri, dans la seconde moitié du XIXe siècle, une contre-culture urbaine à valeur d'opposition politique, religieuse et savante. Dans cet ensemble foisonnant de réélaborations de textes d'origines éparses, l'écart distinctif que revendiquent alors les adeptes d'une «Haute Maǵie » consiste à se réclamer de traditions intellectuelles et religieuses occidentales oubliées ou disqualifiées, et redécouvertes à travers une pratique de collectionneur de livres anciens et d'un savoir bibliophile acquis auprès de libraires-éditeurs spécialisés. L'écriture érudite qui accompaǵne l'édification de prestigieuses bibliothèques privées entend à la fois réconcilier la science et la foi et rétablir une hiérarchisation entre corpus textuels du passé, traductions contemporaines et productions populaires de «grimoires ${ }^{1} »$. L'idée d'un savoir perdu de l'Antiquité ou de l'Orient, en tout cas d'avant le christianisme, hante la modernité qui associe cette perte à tous les livres prohibés par l'Éǵlise où la culture humaniste rêvait de langue parfaite et de paix universelle.

L'exercice que je propose ici n'est pas une nouvelle critique des présupposés idéologiques sous-jacents à l'autonomisation, par quelques historiens des religíions, d'un champ d'études académiques. On le sait bien, faire l'histoire des «courants ésotériques » en affirmant la continuité sur plusieurs siècles de traditions intellectuelles margínalisées par les savoirs dominants équivaut à identifier, de manière arbitraire, des invariants lus en clé phénoménologique. Davantage qu'une histoire culturelle plus rigoureuse dans le choix de ses instruments conceptuels, il s'agit de restituer à la description ethnographique des pratiques distinctives, alors qualifiées de «sciences occultes», liées à une surabondance de textes et de livres, mais larǵement invisibles aux yeux d'observateurs familiers des sociétés de l'imprimé. 


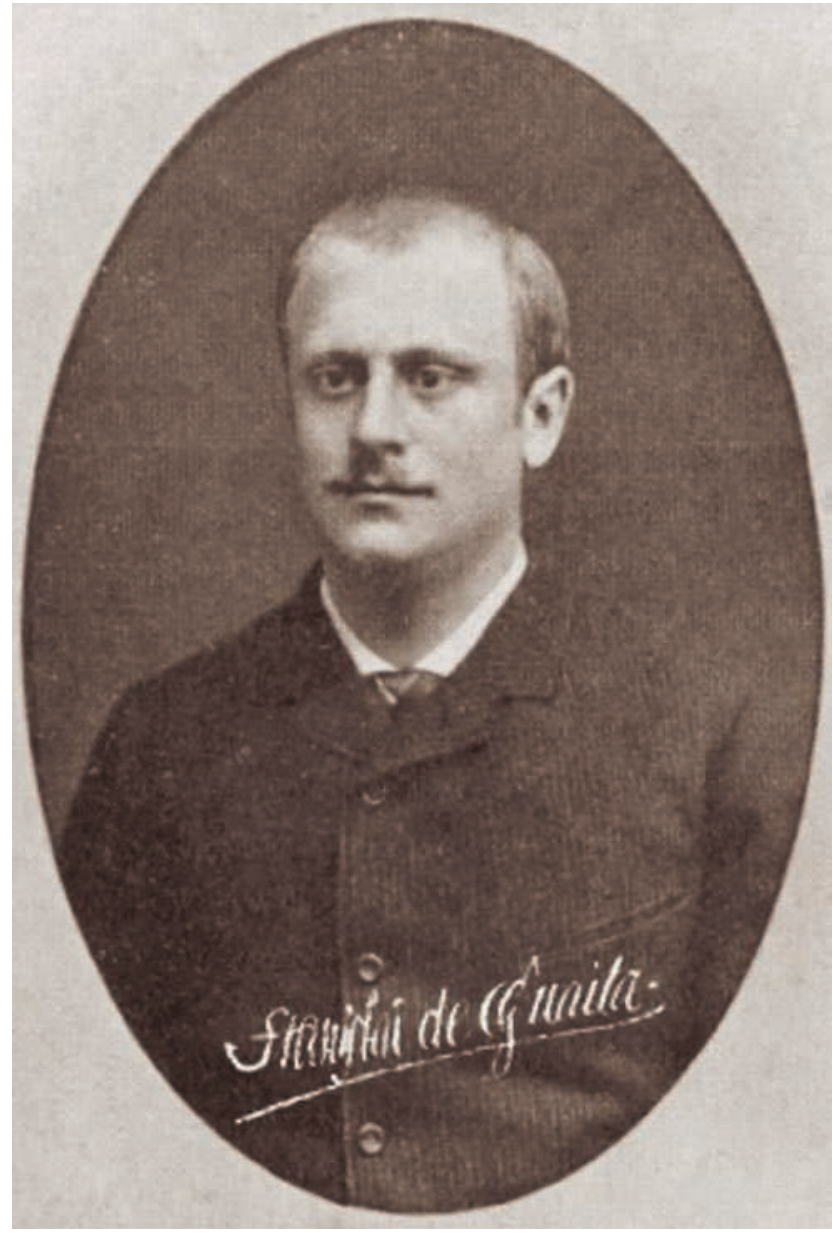

Stanislas de Guaita à 19 ans en 1880. Matteo Omied / Alamy Stock Photo.

Pour les faire apparaître, il faut parcourir les constellations de relations qui mobilisent, autour de quelques figures de "mages» au sens que ce terme a pris chez les écrivains romantiques, plusieurs cercles d'amis, d'institutions, d'érudits et de libraires-éditeurs ${ }^{2}$. Je me limiterai ici à l'une des plus représentatives de ces figures: l'écrivain Stanislas de Guaita (1861-1897), qui se lie aussi bien avec le médecin Gérard Encausse, alias Papus (1865-1916), qu'avec l'écrivain et critique d'art Joséphin Péladan (1858-1918). Tous les trois ont pour maître Éliphas Lévi (1810-1875), le maǵe de la génération précédente, et pour garant l'écrivain et philologue Fabre d'Olivet (1767-1825), qui a fixé le mythe de l'hébreu comme langue primordiale de l'humanité. De famille aristocratique, le jeune Stanislas de Guaita a abandonné une vocation poétique pour devenir la mémoire et l'interprète de savoirs disqualifiés qu'il assimile à une part maudite de la raison
- les «sciences maudites »-, à partir de l'édification d'une très précieuse collection de textes renaissants et modernes. Gérard Encausse, médecin d'origine populaire rallié à la théosophie, est un polyǵraphe prolifique qui hante la Bibliothèque nationale de France pour nourrir sa revue L'Initiation, publier d'innombrables traités et dispenser son enseignement au sein de multiples sociétés. Alphonse-Louis Constant, lui aussi d'oriǵine populaire, a d'abord abandonné sa charǵe ecclésiastique pour fréquenter les milieux saintsimoniens - il est bien connu des historiens du socialisme -, puis il a hébraïsé son nom pour devenir, en tant qu'Éliphas Lévi, le prophète d'une rénovation spirituelle. Chacun d'eux délimite son domaine, forge sa science propre à partir de choix et d'intérêts différents, mais avec des techniques intellectuelles similaires. Ils partagent le même souci de réappropriation du passé de tous les livres, manifestent la même passion pour
2. Sur la double dimension d'universalité, étrangère aux religions particulières, et de primitivisme que ce terme acquiert chez les poètes et écrivains romantiques, voir: Bénichou 1988 


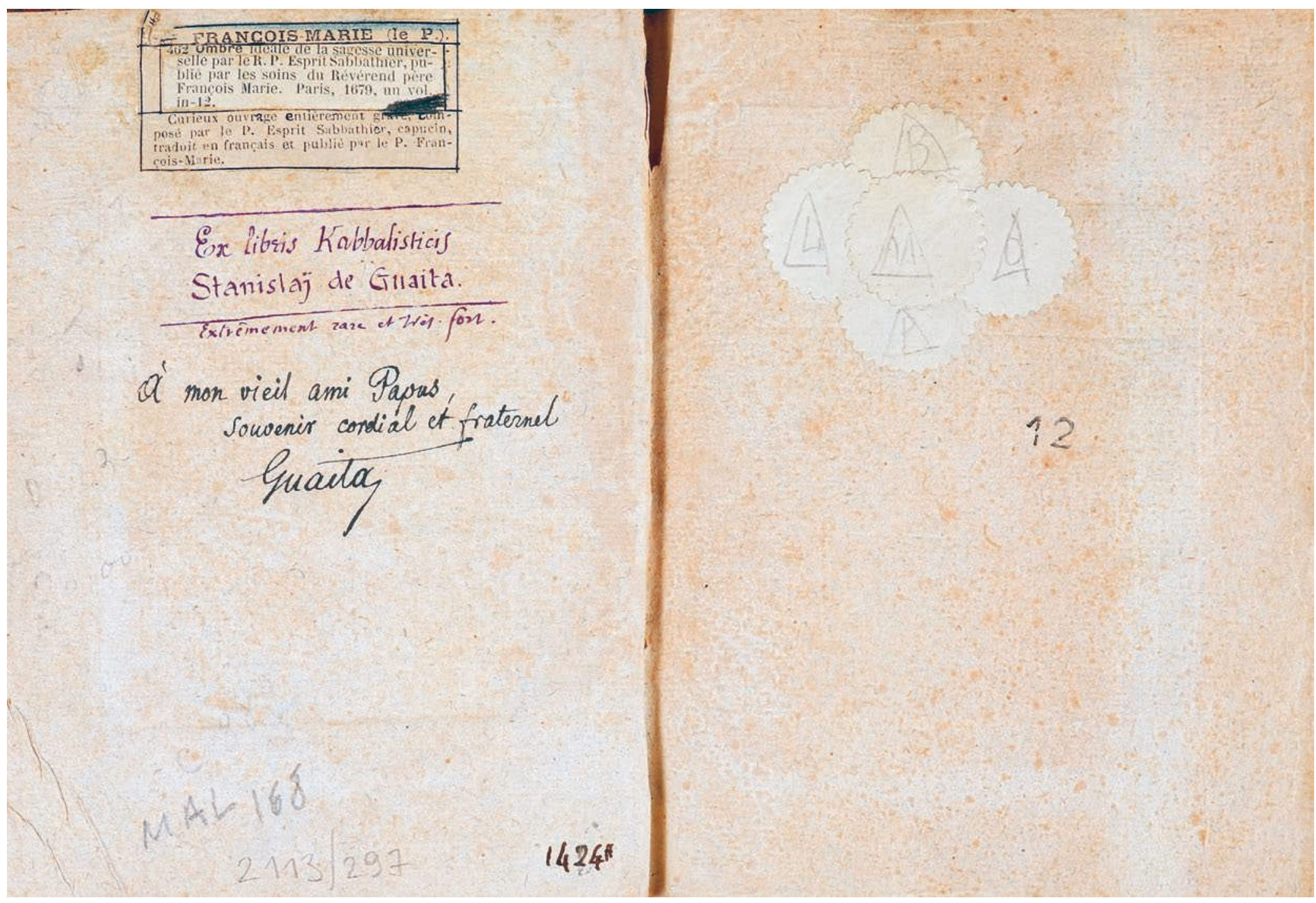

Ex libris et dédicace de Stanislas de Guaita dans le livre Ombre idéale de la sagesse universelle, du R. P. Esprit Sabbathier, Paris, Chamuel 1897. Avec l'aimable autorisation de la Galerie Kâ-Mondo, Paris. 
la diversité des siǵnes et des codes graphiques, et se posent en fondateurs de multiples ordres et sociétés secrètes.

Dans la continuité de l'analyse des inventions rituelles introduites, depuis le début du XIX siècle, sous l'autorité de «la Science», penser par cas nous permettra de rétablir la dimension concrète de formes de vie placées sous le signe distinctif de la «Haute Magiie»; d'identifier les modalités de recomposition de rôles rituels en attente d'interprétation; d'interroger les registres expressifs et les déplacements dans la littérature de cette forme paradoxale de primitivisme reliǵieux.

\section{LE MAGE BIBLIOPHILE}

Issu, par son père, d'une famille noble d'origíne lombarde et, par sa mère, d'une famille lorraine, Guaita fait ses études au lycée de Nancy, où il s'est lié d'amitié avec celui qui deviendra l'idéologue de l'extrême droite française, Maurice Barrès. Étudiant en droit à Paris, «Guaita Stani » se veut poète à la manière de Baudelaire, en consommant force morphine et cocaïne. C'est un roman, Le Vice suprême, où s'affirme comme écrivain le jeune critique d'art Joséphin Péladan, qui lui fait découvrir un art et une science: l' «hermétisme».

L'irruption de ce néologisme, dans la seconde moitié du XIX ${ }^{\mathrm{e}}$ siècle, s'appuie sur la redécouverte historique et philologique du Corpus Hermeticum, dont l'autorité avait été mise à mal par la critique philologique du XVII ${ }^{\mathrm{e}}$ siècle. À l'encontre de la légende faisant d'Hermès Trismégiste un personnage historique contemporain de Moïse et d'Abraham, le philologue Isaac Casaubon montrait, au début des années 1700 , que ces textes liés à la philosophie gréco-égyptienne élaborée à Alexandrie n'étaient pas antérieurs aux premiers siècles de l'ère chrétienne. Tout comme à Berlin, où paraissent en 1854 les éditions scientifiques de l'hermétisme philosophique, Louis Ménard, l'ami de Baudelaire, s'emploie en France à soustraire ces œuvres à l'accusation de faux en les assimilant, dans une perspective d'histoire comparée des religions, à diverses tendances mystiques qui traversent l'antiquité tardive et les premiers siècles de notre ère. Les textes qu'il traduit en «païen mystique» assurent la transition entre la Grèce polythéiste, l'Éǵypte panthéiste et le monothéisme chrétien (Ménard 1866).

Le récit de Péladan se termine par l'évocation, en clé néomédiévale, de la bibliothèque du vieux rabbin Sichem où se retrouvent les deux héros masculins, le jeune mage Mérodach ${ }^{3}$ et un dominicain qui a résisté à toutes les entreprises de séduction féminine:

Des vitrines de pierres gnostiques, des cylindres sumériens; des graphites mystérieux; des panoplies de verges d'Aaron, de fourches de coudrier, d'épées évocatoires; des armoires d'abroxas et des bibliothèques où tenait tout l'œuvre occulte depuis le plus lointain des incunables jusqu'aux rituels d'Éliphas Lévi. Les Séphers, les Targumin, les Talmuds, les Grimoires et les Clavicules étaient là, avec les livres des Mystères d'Elkena que l'on croyait perdus. Des papyrus, des peaux d'onagre burinées au stylet, des lames de pierres précieuses couvertes de hiérogrammes étaient sous verre: musée et bibliothèque du hiéroglyphe et du mystère.

(Péladan 1884: 384).

Pour s'identifier à un maǵe version fin de siècle, il convient de transformer cet exotisme littéraire en la solide érudition qu'exige la construction d'une mémoire culturelle au service d'une utopie sociale. En une quinzaine d'années, l'écrivain rassemble l'une des plus importantes collections européennes de «sciences occultes », donnant naissance à une bibliothèque privée qui a joué un rôle central dans la définition identitaire d'un champ de savoirs et d'une communauté de disciples, mais qui fut dispersée après sa mort. Le souvenir de ce trésor perdu se transmet aujourd'hui sur les sites consacrés à la bibliophilie: en janvier 2014, le bloǵ du site Bibliophile.com publie une enquête à première vue bien documentée sur les difficultés de dénombrement et de classement (Coxe 2014). Faut-il retenir deux mille deux cent vingt-sept livres comme le voulait le catalogue édité par le libraire Dorbon, après la mort de l'écrivain? Ou bien deux mille deux cent trente-cinq si l'on compte les volumes en double? Ou encore deux mille quatre cent soixante-quatre si l'on distingue les traités cousus ensemble? Faut-il distinguer deux ou trois bibliothèques, selon que l'on prend en compte celle de Paris, celle du château d'Alteville ou, encore, celle de «la Chambre de direction du Conseil suprême de la Rose-Croix», la société secrète fondée par l'écrivain? Quelle grammaire a présidé à l'accumulation de tous ces ouvrages, manuscrits ou imprimés? Oswald Wirth, le secrétaire qui établit un premier inventaire, distinguait six catégories qui recoupent, peut-être, leur distribution matérielle ${ }^{4}$. Aujourd'hui, on tend à défaire cette architecture, mais plus on cherche des critères rationnels de classement, plus s'accroît un irrémédiable sentiment d'incomplétude. Il y a les livres qui échappent à l'inventaire car ces objets mobiles s'échangent, se revendent, se donnent au gré des ressources du marché et des déplacements d'intérêt du collectionneur. Il y a les livres qui, à travers plusieurs ventes entre décembre 1898 et juin 1899, se trouvent disséminés dans le monde entier et échappent encore à leur redécouverte. Une dispersion, disent certains, voulue par la famille pour se protéger de leur pouvoir maléfique et priver les occultistes de leur instrument de travail. Enfin, il y a les «faux Guaita », car ce démembrement a donné naissance, comme pour les reliques, à la fabrication de faux ex-libris qu'il faut apprendre à discerner.

Surprenante mélancolie que celle des bibliophiles contemporains, puisque l'on doit à René Philipon, directeur de la «Bibliothèque rosicrucienne » chez le libraire-éditeur Henri Chacornac, un catalogue
3. Passionné d'assyriologie, Joséphin Péladan «babylonise» son nom pour se faire lui-même appeler «Sâr Mérodach Péladan».

4. Ce sont: 1) religion, philosophie, mysticisme; 2) alchimie, hermétisme, kabbale; 3) magie, occultisme, magnétisme, spiritisme, théosophie; 4) divination astrologie, prédictions, chiromancie; 5) sorcellerie, démonologie, magie noire; 6) histoire, archéologie, divers. 
5. «ll passait cinq mois de l'année dans un petif rez-de-chaussée de l'avenue Trudaine, où il ne recevaitque quelquesoccultistes, et don il lui arrivait de ne pas sor pendant des semaines. Il avait amassé là toute une bibliothèque étrange

et précieuse, des textes latins du Moyen Âge, des vieux

grimoires chargés de

pentacles, des parchemins

enluminés de miniatures, des traités d'alchimie, les éditions les plus estimées de Van Helmont, Paracelse, Raymond Lulle,

Saint-Martin, Martinès de

Saint-Martin, Martinesse

Pasqually, Corneille Agrippa,

Pierre de Lancre, Knorr de

Rosenroth, des manuscrits

d'Éliphas, des reliures signées

Derome, Copé, Trauzt-Bauconnet,

Chambolle-Duru, des ouvrages

de science contemporaine.

(Barrès 1898: 29)

6. Il s'agit de Heinrich Khunrath, voir infra. de plus de trois cents pages, Stanislas de Guaita et sa bibliothèque occulte (1899), qui permet de prendre la mesure de tous les savoirs convoqués pour construire l'espace matériel et le dispositif intellectuel qui ériǵent, en cette fin de siècle, la personne du maǵe en expert des «sciences maudites»:

Les ouvrages les plus rares sur l'alchimie, la magie, l'illuminisme, il les possédait tous en des éditions princeps, grands de marges, intacts, toujours revêtus d'une reliure adéquate au contenu du volume, non par manie de bibliomane, loin de là, mais, parce qu'artiste et savant avant tout, il estimait que la forme devait être digne de la pensée.

(Philipon 1899: VI)

Ce portrait du mage en bibliophile met l'accent sur le premier renversement qu'opère cette nouvelle figure sociale: réhabiliter la passion pour le livre ancien, dont les excès ont été férocement moqués tout au long du XVIII ${ }^{\mathrm{e}}$ siècle sous le nom de «bibliomanie », cette maladie du lettré qui fait son apparition dans La Nef des fous de Sébastien Brant (Desormeaux 2001: 23). Il fixe le bon usage des livres en répondant à la disqualification d'une «passion ridicule», de l'avis de D'Alembert, qui fait accumuler des livres sans les lire pour affirmer un statut social ou pour accéder à la totalité du savoir en faisant l'économie des médiations nécessaires.

Objet précieux et instrument de connaissance, le livre conserve, sur ses paǵes de garde emplies d'annotations, la trace des manières de lire d'un gardien des livres et des textes. Le catalogue, qui les reproduit, nous permet de regarder travailler l'érudit qui détaille les raisons historiques de la rareté d'un exemplaire ou d'une édition, rédige de longues notes bibliographiques, esquisse une analyse critique, compare les traductions ou bien condense en une phrase l'apport intellectuel d'un ouvrage. Autant de formes de réécriture qui permettront de produire de nouveaux textes et d'affirmer des choix idéologiques. Ainsi, les nombreux traités de démonologie portent la trace de son aversion pour la violence des inquisiteurs. Sur un très rare exemplaire du traité d'Henri Boguet, il note:

Entre les plus féroces démonologues de son temps, H. Boguet se distingue par son atrocité naïve, doublée d'une imperturbable sottise... La famille de Boguet a dépensé des sommes considérables à racheter, pour les détruire, les exemplaires de son ouvrage, ce qui explique la rareté des différentes éditions.

(Philipon 1899: 12)

De plus, Stanislas de Guaita fait du livre lu, commenté, parfois réécrit, l'instrument du lien social, au sein d'une communauté de jeunes gens qui semble ressusciter les académies de sagesse antique en régime renaissant, où la transmission d'un discours problématique exige de passer par l'oralité, d'être adressée à chacun à travers le souffle de la voix. En contraste avec l'imaginaire faustien qui fait les délices d'un Péladan, des idéaux d'harmonie sociale rassemblent, chaque jeudi soir, quelques disciples réunis dans une sociabilité d'initiés ${ }^{5}$, pour écouter le maître, vêtu de rouge, argumenter livre en main et restituer aux frères du passé la séduction de la parole vive, en leur prêtant son corps et sa voix :

Il fallait l'entendre soutenir la thèse de l'objectivité, étayant son argumentation sur maints auteurs favoris, principalement sur Khunrath ${ }^{6}$, dont il possédait un exemplaire magnifique, riche de ses douze planches intactes, annoté d'un bout à l'autre de sa propre main... Des phrases qui, jusqu'alors, avaient passé inaperçues à nos yeux, prenaient, proférées par sa voix au timbre clair qui rythmait avec une conscience certaine, une signification nouvelle et inusitée.

(ibid.: VI)

Les disciples qui lui rendent hommaǵe, après sa mort, dans un numéro spécial de la revue L'Initiation, retiennent l'égale attention portée à déchiffrer textes et figures (Jollivet-Castellot 1998).

\section{LA PRIMITIVE SYNTHÈSE}

Quelle grille de déchiffrement, quel travail d'appropriation le maître met-il en œuvre? Quel réseau d'autorités informe ses manières de lire et d'écrire? Des divers volumes des Essais de sciences maudites, publiés entre 1890 et 1897 par l'éditeur scientifique Georges Carré, retenons le premier - Au seuil du Mystère - , qui est en fait une visite guidée de la partie la plus précieuse de cette bibliothèque destinée à construire une parenté savante sur le mode d'une lignnée unifiée de mages, à partir d'auteurs venus d'horizons intellectuels dissemblables. Identifier les pratiques de lecture adoptées par Stanislas de Guaita nécessite, cependant, de les confronter aux horizons conceptuels dans lesquels les historiens des sciences inscrivent, aujourd'hui, les livres et les savoirs convoqués. Le fonds ancien rassemble les principaux textes qui commencent à affirmer, à partir de la Renaissance, l'affinité des diverses traditions philosophiques et religieuses néoplatonisme, astrologie, hermétisme, alchimie, kabbale -, liées au mouvement antérieur des traductions arabo-latines et hébraïco-latines des livres de magie. Soit les textes de ce que l'on a appelé l'occultisme de la Renaissance, fondé sur des modes de pensée et des exiǵences expressives margínalisées par la science cartésienne; de par leurs somptueuses gravures, ces livres sont désormais perçus comme des joyaux de la production typographique et des arts graphiques d'Ancien Régime. Le mage harmonise ces diverses traditions intellectuelles en assimilant la mise en ordre des textes à une mise en ordre cosmique. La bibliothèque mentale qu'il invite à parcourir situe les textes alchimiques sur le plan naturel ou sensible, les arts 
divinatoires (astrologie, chiromancie) sur le plan moral ou psychique, les textes de la kabbale sur le plan divin. Placé en position de novice, le lecteur doit apprendre à lier ensemble ces trois registres en faisant s'entreinterpréter textes et figures.

Deux gravures de la seconde édition de l'Amphithéâtre de la sagesse éternelle de Heinrich Khunrath, ô combien admiré, introduisent le novice «au seuil du mystère». Elles ont été reproduites à partir d'un exemplaire de l'édition complétée de splendides gravures en taille-douce parue à Hanau en 1609, qui est en la possession de Guaita ${ }^{7}$. Sans doute a-t-il commenté la gravure donnant à lire comme un paysage que l'on parcourt La Table d'émeraude ${ }^{8}$, cet enseignement sous forme de formules allégoriques que, dans une variante du savoir révélé, Hermès Trismégiste a laissé aux hommes, gravé sur un rocher à l'emplacement de son tombeau, dit la légende ${ }^{9}$. Mais, pour prendre place dans la lignnée d'Hermès, il inscrit son propre enseignement sous le signe du Grand androgyne hermétique et de la Rose-Croix pentagrammatique, soit la première et la quatrième figures.

Alors que la critique historique range Heinrich Khunrath parmi les auteurs de la tradition alchimique, la «plume kabbalistique» du mage commente ce "résumé hiéroǵlyphique de toute une doctrine» en le rattachant aux grands thèmes du mouvement intellectuel que l'on nomme la «kabbale chrétienne», exposés dans une écriture émaillée de lettres hébraïques et de quelques figures géométriques ${ }^{10}$. Placé sous les auspices de Khunrath, ce premier volume de «sciences maudites » se présente, alors, comme une sorte de manuel introductif à une tradition mystique qui s'emploie à ensauvager les énoncés dogmatiques de la culture chrétienne: c'est «une langue à apprendre », assure le mage, pour atténuer la désorientation du lecteur novice (Guaita 1890: 123). La vénération portée au «style âpre, exalté, presque barbare» de Khunrath, aux «rocailles de la forme», au «tumulte épique des vocables» fait dès lors de Guaita le principal artisan de la redécouverte au XIX siècle d'imaǵes de l'alchimie historique, lues en clé kabbalistique, pour inventer cette «primitive synthèse »: autant dire ces lectures métaphoriques que les historiens des sciences appellent, aujourd'hui, à mettre à distance pour retrouver les savoirs médiévaux sur les propriétés de la matière que l'on nommait, alors, indistinctement alchimie ou chimie (Principe et Newman 2001; Kahn 2007).

Lire une figure comme un «résumé hiéroglyphique» participe d'interrogations sémiotiques héritées de la Renaissance, sur les vertus respectives des écritures alphabétiques et logographiques auxquelles la Haute Maǵie fait retour. Dans la bibliothèque du mage, figurent en bonne place deux exemplaires du Mutus Liber, considéré comme le principal recueil de philosophie alchimique du XvII ${ }^{\mathrm{e}}$ siècle. L'un est un objet composite: un exemplaire de l'édition originale parue à La Rochelle en 1677, exclusivement composée de quinze figures, a été relié avec un moderne «livre muet» dessiné en $1824^{11}$. L'expression qui a traversé les siècles désigne une forme d'écriture par l'imaǵe - «écrire par figures»-, qu'il convient de rattacher à l'intérêt pour l'Égypte et ses écritures que les humanistes appréhendaient à travers le courant égyptophile grec incarné par Horapollon. On attribue à ce philosophe du ve siècle, réfractaire au christianisme, le seul traité sur les hiéroglyphes qui nous soit parvenu de l'Antiquité, les Hieroglyphica ${ }^{\mathbf{1 2}}$, dont un manuscrit retrouvé en 1419, puis édité en 1505 à Venise, a relancé le questionnement sur l'apparence figurative des hiéroǵlyphes, au détriment de toute valeur phonétique, autant dire sur l'intérêt et les difficultés

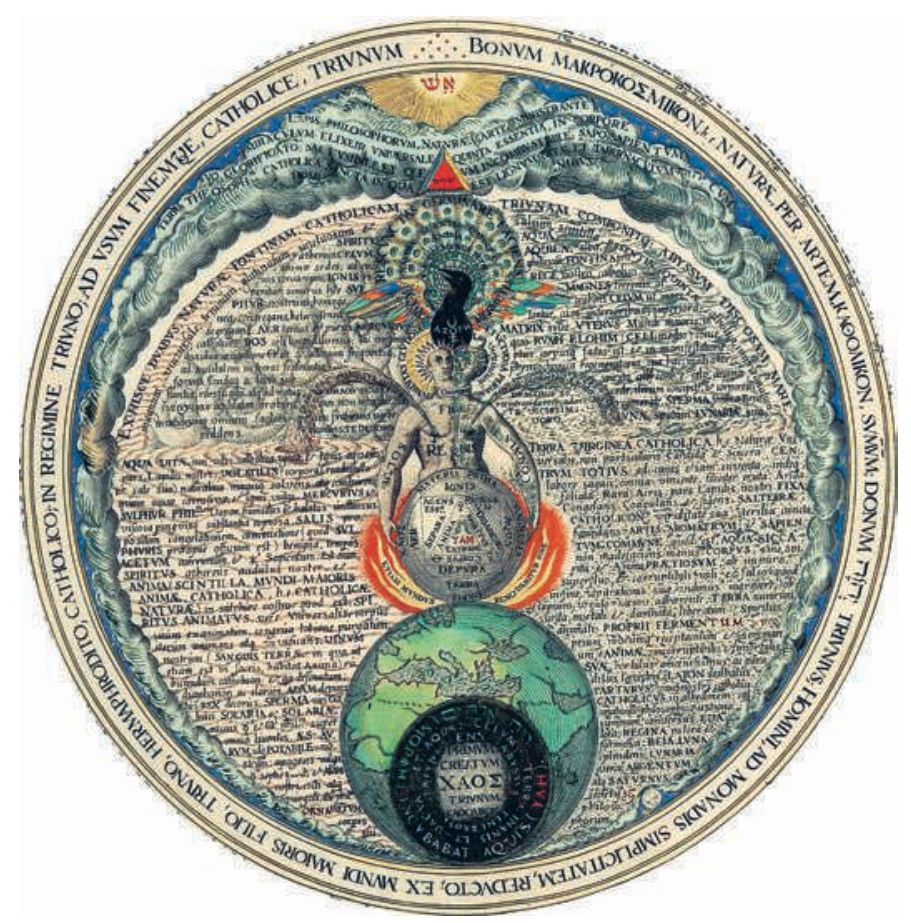

Rebis, L'Homme microcosme comme Adam-Androgyne, et l'oiseau d'Hermès, gravure de Hans Vredeman de Vries publiée dans Amphitheatrum Sapientiae Aeternae de Heinrich Khunrath, Hambourg, 1595. L'écriture, qui mêle caractères latins, grecs et hébreux, est, elle-même, traitée comme une matière picturale. Heritage Images / Fine Art Images / akg-images. 


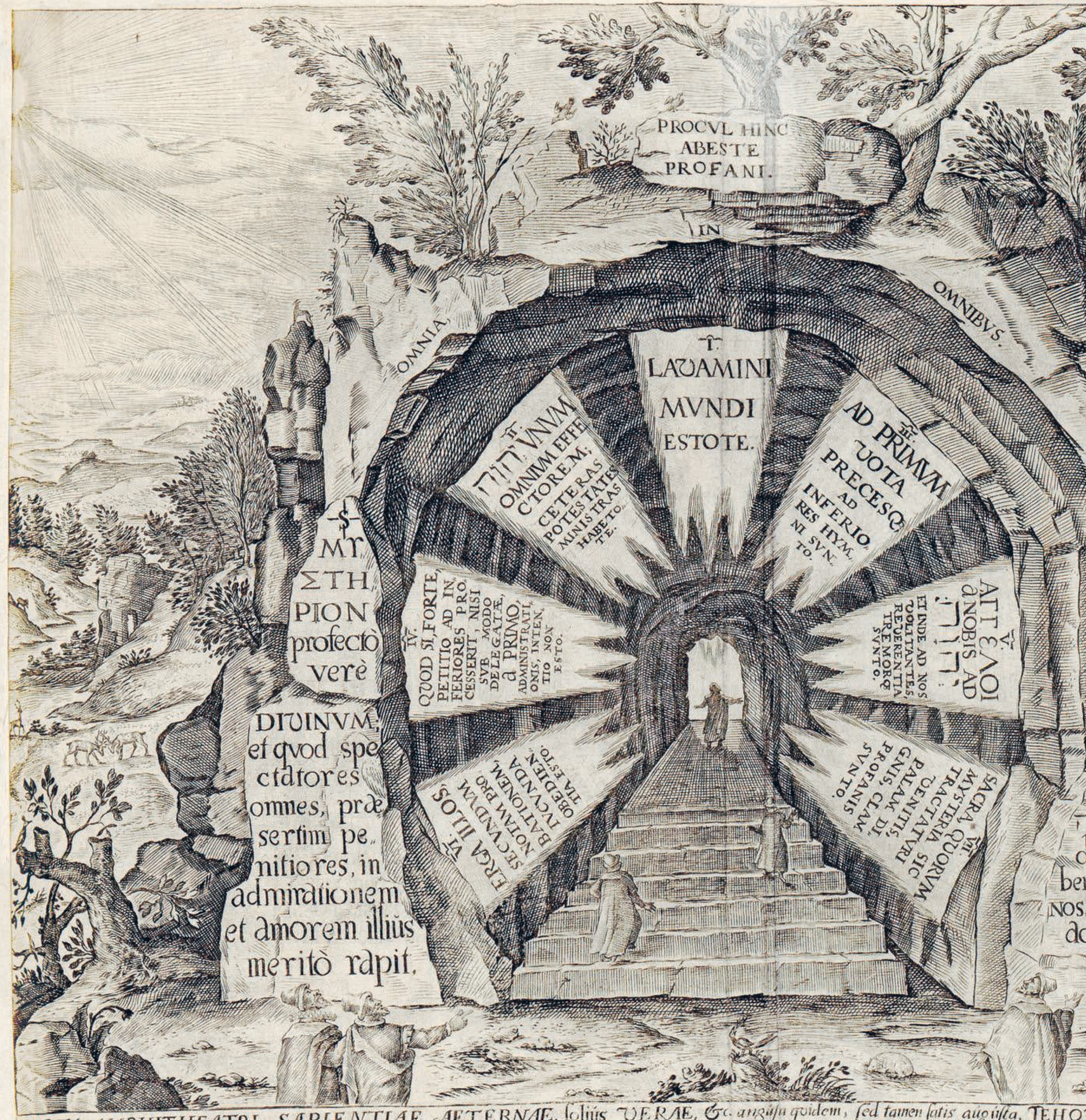

DORTA AMPHITHEATRI SAPIENTIAE AETERNAE, COlius UERAE, Gc. ansuja quidem, fed tamen fatis aug iffa, TEHO fiam myfticam, prologetican nimirum illam, fibi prefixam, picturá hic fignificatam, graduüm lepiem Theofophicorum, veré Philofophicam,

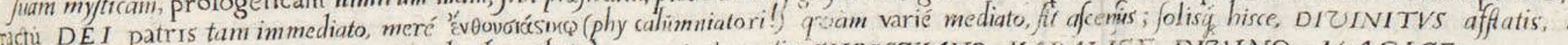
wié portam corüfcantes, copia introgredicndi, ocülisq \& corporis ó mentis CHRISTIANO-KABALICE, DIVINO - M A GICE, nec non

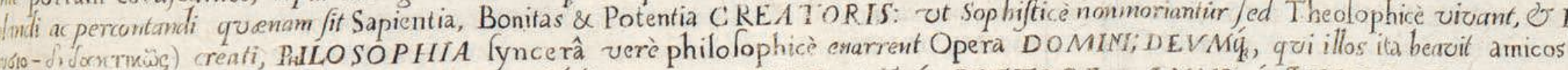
KHVNRATH LIPS. Theolophix amalore fideli, \& UMEDICINAE vtriúsú; DOCTORE. ANNO á JHSTH CHRISTO 


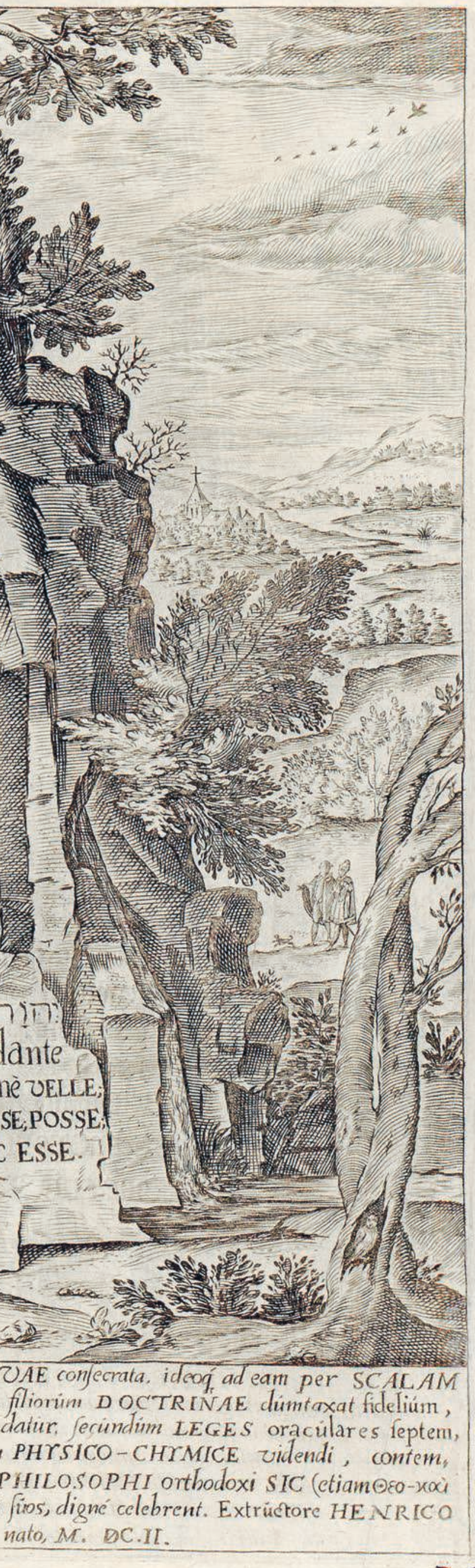

Porte de l'amphithéâtre. Gravure de Amphitheatrum sapientiae aeternae, 1595 de Heinrich Khunrath (vers 1560-1605). II s'agit de l'une des neuf gravures de la seconde édition de l'Amphiteatrum sapientae aeternae publiée après la mort de Heinrich Khunrath. Elle montre les théosophes qui, à la différence des savants de l'université, pénètrent dans I'Antre de la Nature, I'un des trois livres de la connaissance avec Dieu et l'expérience personnelle de l'alchimie. BU Santé AJG, Université Toulouse III - Paul Sabatier, Res Med XVII D104971. 
12. Sur les étapes du déchiffrement des hiéroglyphes égyptiens, voir la mise au point de Farout 2016. Pour un renouvellement de la figure d'Horapollon, voir le récent colloque: «Horapollon hellénisme et hiéroglyphes dans I'Antiquité tardive», organisé par Jean-Luc Fournet, Collège de France, 13 juin 2018: en particulier, la communication d'Andreas Stauder, «Les faces multiples du signe graphique égyptien».

13. Henri Khunrath, Amphithéâtre de la sagesse éternelle seule vraie, chrétienn et kabbalistique, divine et magique, physique et chimique dans son universelle tri-unité, texte complet traduit pour la première fois en français, Paris, Chacornac, «Bibliothèque rosicrucienne», 1900

Traduction du latin de Papus et Marc Haven.

14. Cette anthologie rassemble des textes comme le Zohar du xIII e siècle et des auteurs regroupés dans la «kabbale de Safed», qui se développe au xvle siècle autour d'Isaac Louria. Elle s'inscrit dans la promotion des hebraic et de leur étude, alors que la rivalité confessionnelle et religieuse est intense. Pour un renouvellement de l'étude de ce mouvement intellectuel et de ses enjeux au sein de la philosophie du xvı" siècle, voir: Vileno 2016

15. L'hébreu qabalah, que I'on traduit par «réception» ou «tradition», désigne la transmission d'un enseignement de maître à disciple; en tant que technique de lecture et d'interprétation de la Torah, son sens se restreint, à partir des xII et xIII ${ }^{e}$ siècles, à la mystique juive avec son corpus fondateur le Bahir et le Zohar; au cours du $\mathrm{xvl}$ siècle, émerge un courant de mise en convergence de la kabbale chrétienne avec le symbolisme alchimique, fondée sur l'homologie entre la réduction du langage à ses lettres élémentaires

et la réduction de la matière à ses éléments. Sur la pansémiotique kabbalistique, voir: Eco 1997 [1994]: chap. II.

16. Le personnage historique de Nicolas Flamel (1330 env.-1418) est un écrivain puv.-1418) est un écrivain
public et libraire-juré parisien.

17. Jeu narratif sur l'étymologie latine qui dérive liber, «livre», de liber, «écorce». à penser la transformation d'images en signes (Eco 1997 [1994] : chap. VII; Christin 1995: 61-64). Assimilés à des dessins symboliques, allégoriques ou métaphoriques, ils composeraient une écriture secrète réservée au domaine de la religion et destinée aux seuls initiés.

Le Mutus Liber participe de la pensée visuelle des livres d'emblèmes, ces formules iconiques issues de l'interrogation sur le devenir signe des images. Il porte à son comble, a-t-on pu dire, la confiance dans le figuratif pour faire d'un répertoire de métaphores visuelles le mode de transmission le plus efficace d'un savoir technique modelé par l'imaǵinaire d'une remontée dans le temps qui ouvrirait l'accès à un savoir originaire (Obrist 2003; Edeline 2009). Qualifier, en cette fin du XIX siècle, les imaǵes de Khunrath de «résumé hiéroǵlyphique » vient, dès lors, intensifier un déplacement déjà présent chez ce disciple de Paracelse condamné par la Sorbonne, qui pensait métaphoriquement les opérations alchimiques pour réanimer la langue de la spiritualité chrétienne (Forshaw 2006). La société rosicrucienne fondée par Guaita entreprend la première traduction française de cet Amphithéâtre de la sagesse éternelle qui paraît en 1900 chez le libraire-éditeur Chacornac ${ }^{13}$.

Le souci de reconstruire la «doctrine intégrale des maǵes » s'appuie, de même, sur un auteur et une œuvre du XVII siècle possédée en plusieurs exemplaires la Kabbala Denudata (La Kabbale dévoilée ou La Doctrine transcendantale, métaphysique et théologique des Juifs), anthologie de textes juifs traduits en latin par le protestant Christian Knorr von Rosenroth (1636-1689) et publiée en deux volumes à Sulzbach en 1677 et 1684. Médecin, philologue, théologien, philosophe: c'est l'un des principaux artisans de cette christianisation de la mystique juive qui consiste à appliquer aux Écritures - Ancien et Nouveau Testament - ses méthodes interprétatives, où le geste apologétique se nourrit de la redécouverte des lanǵues antiques et orientales - hébreu, grec, égyptien.

Lanthologie de Knorr von Rosenroth inclut des traductions latines d'ouvrages classiques, des lexiques, des extraits de l'abondante correspondance que l'auteur entretenait avec le philosophe Henry More et l'alchimiste François-Mercure Van Helmont. Seize figures, souvent reproduites, complètent cette encyclopédie ${ }^{14}$ qui faisait transiter un héritage intellectuel vers un autre univers religieux et qui constitue, jusqu'à la fin du XIX ${ }^{\mathrm{e}}$ siècle, la source principale de la littérature non juive sur la kabbale ${ }^{15}$. Le mage moderne s'est, à son tour, inscrit dans la chaîne des scripteurs juifs en complétant de sa main un exemplaire inachevé, tenu dès lors pour le plus précieux. Il a également inséré une «figure hiéroglyphique» réalisée par son secrétaire dans ce style néogothique qu'affectionne le médiévalisme fin de siècle, pour donner à voir le «mystère » du Beraeshit, ce premier mot de la Genèse que Fabre d'Olivet proposait de traduire par «premièrement-en-principe» à partir des trois sens du substantif raesh - tête, chef, principe : surǵissant d'une coupe remplie d'eau, trois visages sont réunis en une seule tête, coiffée d'une tiare en forme de tour surmontée d'une croix (Philipon 1899: 73).

Cette toute moderne «primitive synthèse» possède, comme il se doit, son livre révélé, qui appartient au légendaire d'Abraham le Juif et de Nicolas Flamel. À l'image d'autres manuscrits que l'on dit transmis par des vieillards ou gardés par des êtres fantastiques, Le Livre d'Abraham le Juif n'a tout d'abord qu'une existence littéraire dans l'autobiographie fictive d'un personnage historique, Nicolas Flamel, appelé à devenir une figure nationale d'alchimiste ${ }^{16}$ :

Les Figures hiéroglyphiques de Nicolas Flamel, ainsi qu'il les a mises en la quatrième arche qu'il a battie au Cimetiere des Innocens à Paris, entrant par la grande porte de la rue $S$. Denys, \& prenant la main droite; avec l'explication d'icelles par iceluy Flamel. Ce texte qui paraît en 1612 se présente comme la traduction d'un récit autobiographique écrit en latin entre 1399 et 1413 , où le narrateur expose comment il a acquis pour deux florins un mystérieux livre, fait de «trois fois sept feuillets d'écorce ${ }^{\mathbf{1 7}}$ » reliés dans une couverture de cuivre «toute gravée de lettres et de figures », et ayant pour titre: Le Livre d'Abraham le Juif, prince, prêtre lévite, astrologue et philosophe, à la gent des juifs par l'ire de Dieu, dispersée aux Gaules, salut. D. I. Texte et enluminures, décrites mais non reproduites, se rapportent au processus du Grand OEuvre que Nicolas Flamel et son épouse, dame Pernelle, s'emploient en vain à réaliser durant vingt et un ans - autant d'années que le livre compte de feuillets -, faute de pouvoir «lire» les images. Le narrateur entreprend alors un pèlerinage à

Saint-Jacques-de-Compostelle où il rencontre un vieux médecin juif converti, maître Canches, qui lui donne le sens du symbolisme des images, avant de mourir. De retour à Paris, Flamel parvient à transmuter du mercure en argent, puis en or, et avec la fortune ainsi acquise, l'alchimiste fonde diverses œuvres pieuses et fait peindre sur une arcade du cimetière des Innocents des figures qu'il commente selon une double interprétation, théologique et philosophique.

Lue comme un traité médiéval, l'autobiographie fictive de 1612 connut un succès immédiat car elle faisait s'entrecroiser plusieurs motifs : l'origine alchimique de certaines fortunes bourgeoises, l'attribution des traités à des autorités antiques ou médiévales, le recours au langage allégorique et aux figures symboliques dans la tradition des livres d'emblèmes, où les imaǵes sont complétées par une brève leçon morale. Le légendaire de Flamel, transmis au siècle suivant sur le mode de la controverse, propose une figure catholique d'alchimiste qui révère la Vierge et les saints, et part en pèlerinaǵe, alors que ce savoir est dominé par les réformés du renouveau paracelsien. Les figures du Livre d'Abraham le Juif, qui relèvent initialement de l'ekphrasis, surgíissent dans des manuscrits postérieurs qui n'ont pas échappé à l'attention de Guaita. L'un, du XvII ${ }^{\mathrm{e}}$ siècle, orné de douze imaǵes semblables, dit-on, à des miniatures de livres d'heure, aurait appartenu à Alberti. Le maǵe a complété le second, du XVIII ${ }^{\mathrm{e}}$ siècle, en ajoutant des écrits de sa main et des comptes rendus de ses essais, ainsi 


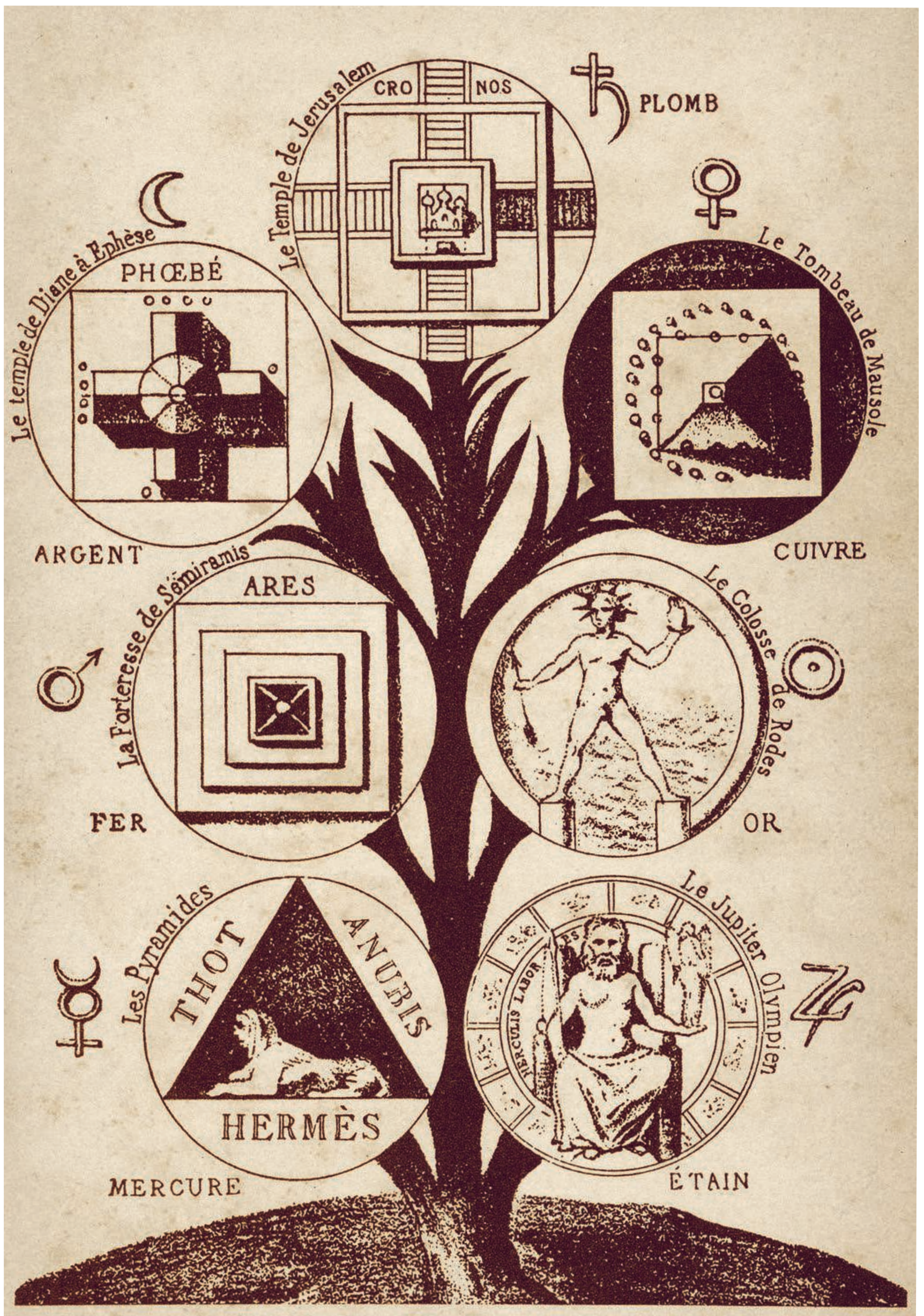

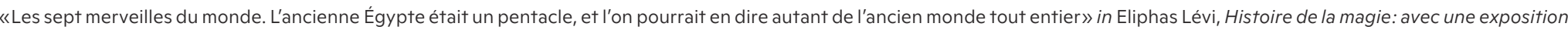
claire et précise de ses procédés, de ses rites et de ses mystères. Paris, G. Baillière, 1856, t. I, p. 173. La figure met en correspondance des traditions religieuses et des codes graphiques al'interieur de la tradition juive de figuration des Séphiroth ou émanations de la puissance créatrice. Akg-images/Fototeca Gilardi. 


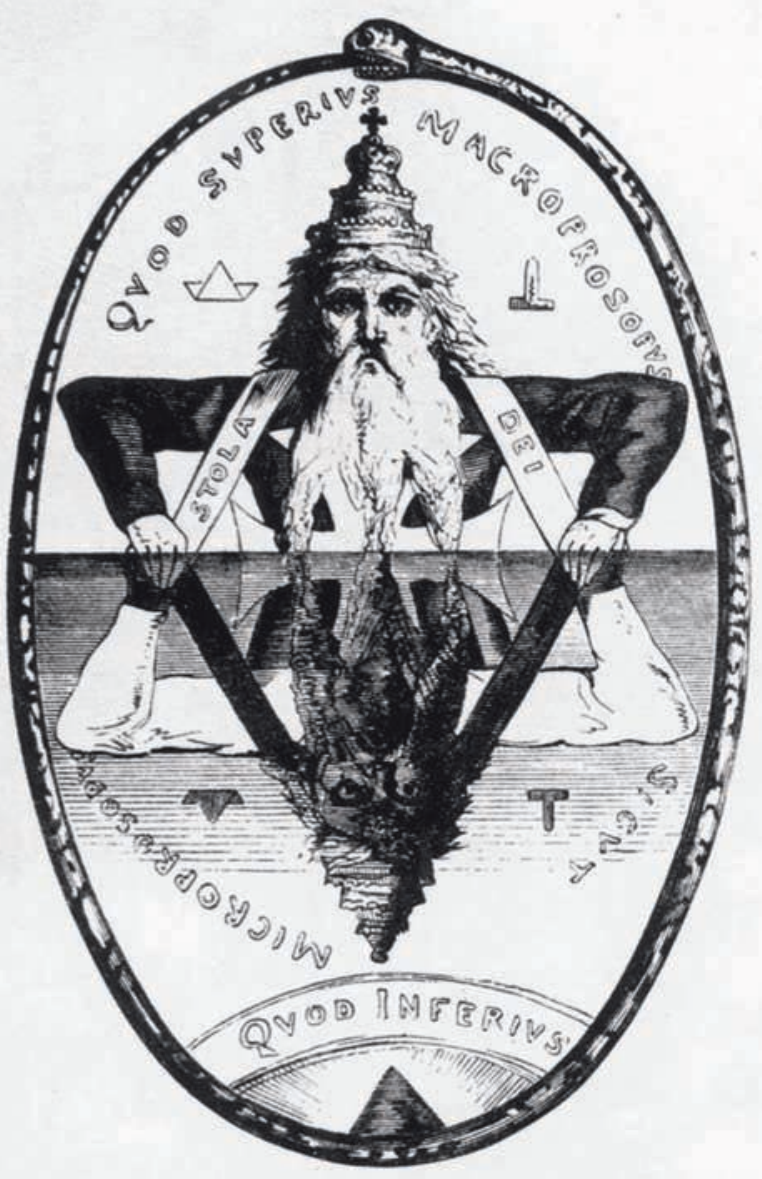

\section{D0GME ET RITUEL}

DE LA

\section{HAUTE MAGIE}

PAL

ÉLIPHAS LÉVI

NOUVELLE EDITION

Avec 24 figures

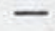

TOME PREMIER

Dogme

\section{PARIS}

LIBRAIRIE GENERALE DES SCIENCES OGC:UI.TER CHACORNAC FRERES

11, QUAI SAINT-MIC:HEI., "I

1930

«Le symbole de Salomon. Le double triangle de Salomon, figuré par les deux vieillards de la cabale; le macroprosope et le microprosope; le Dieu de lumière et le Dieu des reflets; le miséricordieux et le vengeur; le Jéhovah blanc et le Jéhovah noir» in Eliphas Lévi, Dogme et rituel de Haute Magie. Paris, Chacornac, 1900, tome I, p. V. Les termes «macroprosope» et «microprosope» - le Grand Visage et le Petit Visage - renvoient à la kabbale juive @ Tallandier / Bridgeman Images. 
que le procès-verbal d'une expérimentation de suggesestion mentale conduite à Nancy avec le Dr Liébaud. À l'image romantique d'une science alchimique incomprise et persécutée, Guaita substitue ainsi celle d'une vénérable continuité où les anciens savoirs transmis à travers une chaîne d'initiés peuvent s'accorder aux plus modernes nouveautés.

Rassembler des éditions originales, annoter de précieux exemplaires, en poursuivre la rédaction et l'illustration, en établir des traductions : telle est l'emprise des livres de haute magie sur celui qui se voulait poète, artiste et savant, pour s'inscrire à son tour dans une succession de scripteurs, maîtres de la diversité et de la conversion des siǵnes. Ainsi peut, concrètement, s'instaurer un lien de filiation avec cet humanisme de l'Occident latin qui entendait commander à la nature par le détour d'entités contrefactuelles - anges, esprits, Dieu - en maîtrisant leurs singulières modalités de communication. Un humanisme qui, pour ce faire, s'efforçait de remonter vers le sens oriǵinel du texte biblique en opposant à l'adaptation historique de son message le «sens caché», autant dire altéré des Écritures, à rétablir à partir des propriétés respectives des langues de traduction. Faire s'entreinterpréter le néoplatonisme hermétique, la kabbale juive et le christianisme impliquait, alors, des spéculations sur les rapports entre lanǵue originaire et diversité linguistique (Eco 1997 [1994]; Simon 2018), sur la motivation des signes et sur l'unité perdue entre parler et écrire, écrire et dessiner. Ce travail spéculatif mobilise une virtuosité graphique qui s'exerçait aussi bien dans la pratique rituelle que dans l'océan des textes produits par cet immense exercice de traduction, en leur donnant cet aspect si singulier qui continue à exercer sa fascination sur les lecteurs modernes. Mais comment remotiver ces spéculations et ces techniques intellectuelles au tournant des $\mathrm{XIX}^{\mathrm{e}}$ et $\mathrm{XX}^{\mathrm{e}}$ siècles?

\section{LA LANGUE DES MYTHES ET DES EMBLÈMES}

Stanislas de Guaita entend rétablir la langue des mythes et des emblèmes contre les innovations reliǵieuses les plus populaires de cette fin du XIX ${ }^{\mathrm{e}}$ siècle, les «divagations spirites» d'Allan Kardec et l'extravagance de Mme Blavatsky, dont, cependant, il admire l'œuvre; et contre la "science officielle», entendons le positivisme et l'historicisme qui dominent le monde académique. Des affinités électives relient l'aristocrate à quelques modernes qui entendent surmonter les divisions religieuses entre les trois monothéismes en même temps qu'ils inventent diverses formes de «fraternités » comme préfiguration d'une société régénérée. À certains, il reprend le terme de «synarchie» pour désigner la forme de gouvernement idéal; à d'autres, leur érudition sur la kabbale; mais c'est, avant tout, Éliphas Lévi, dont l'encyclopédisme parcourt tous les savoirs à réunifier, qui est reconnu comme maître, autant dire comme créateur de modèles, pour «retrouver le mot perdu des anciens mystères » (Guaita 1890: 51).
Que faire, tout d'abord, de l'irritante opacité graphique du «verbe hiéroglyphique» qui a contribué à dissocier vérité et spiritualité?

La vue d'ensemble y contribuait: signes crochus des planètes, lettres hébrä̈ques des hiérogrammes, caractères arabes des grimoires, hautes fantaisies apparentes des pentacles et bizarrerie mystique des paraboles; toutes choses superlativement diaboliques au sentiment des idiots et des ignares, puériles à première oue au gré des esprits logiques; irritantes, en tout cas, pour la curiosité de chacun. De tout temps, les sages avaient parlé la langue des mythes et des allégories, mais jamais l'obscurité de la forme ne se dut plus mystérieusement épaissir qu'au Moyen Âge et jusqu'au siècle dernier... (ibid.: 20-21)

Les modernes artisans des sciences occultes s'empressent d'attribuer cette opacité à la censure religieuse, et Guaita partage ce point de vue, aujourd'hui mis à mal par les recherches conduites sur la très sophistiquée culture graphique dont participent les arts magiques présents dans l'Occident chrétien entre les $\mathrm{XII}^{\mathrm{e}}$ et $\mathrm{XV}^{\mathrm{e}}$ siècles. Nous connaissons mieux, depuis une vingtaine d'années, les sources d'inspiration de ces compléments visuels des invocations et des exorcismes adressés aux anges, aux esprits, aux démons, dont l'efficience ne passe ni par la parole ni par la compréhension; nous connaissons mieux le répertoire et les contaminations des écritures occidentales utilisés par les rédacteurs de traités ainsi que les horizons linguistiques, les motivations culturelles et les champs sémantiques auxquels il convient de les rattacher (Grévin et Véronèse 2004; Boudet 2006). Siǵnes astraux, signnes dérivés de l'idéographie chrétienne, signes qui évoquent les idéogrammes extrême-orientaux, signes géométriques, lettres des alphabets latin, grec, hébreu pensés comme ensemble linguistique renvoyant aux langues de la révélation, alphabets secrets et écritures inventées, techniques de sténographie et alphabets exotiques: nombreuses sont les ressources scripturaires mobilisées pour instaurer une relation contractuelle avec des entités non humaines ${ }^{\mathbf{1 8}}$. Mais comment réinventer, au XIX ${ }^{e}$ siècle, cette culture graphique liée à la quête d'un savoir primordial?

Dans le mouvement de redécouverte des textes de la kabbale, prenant appui sur l'œuvre canonique de ce corpus - le Sefer ha Zohar ou "Livre de la splendeur» de Moïse de Léon, qui rassemble les thèses des écoles castillanes médiévales sous la forme d'un commentaire des Écritures saintes -, Guaita s'exerce à conduire de nouvelles démonstrations selon les techniques de la kabbale numérique ${ }^{19}$. Mais, sur le versant visuel, il s'en remet au travail figuratif d'Éliphas Lévi qui recrée «clavicules», «pentacles» et «arcanes» tout en incitant son disciple Oswald Wirth à en poursuivre la réalisation ${ }^{20}$. De fait, Éliphas Lévi, que l'on appelait simplement l'«artiste» dans son quartier
18. Je suis ici la belle analyse développée par Benoît Grévin et Jules Véronèse (2004). Pour les langues occultes de la Renaissance, voir: Béhar 1996

19. Sur la canonisation du Zohar, voir: Idel 1998: 422-425. Les nombres étant représentés par des lettres, on peut calculer la valeur numérique d'un mot puis construire des analogies à partir d'autres mots ayant la même valeur numérique.

20. Oswald Wirth dessine et édite en 1889 un premier ensemble de vingt-deux arcanes à partir d'une recherche iconographique à la Bibliothèque nationale et des commentaires de Stanislas de Guaita, qui I'incite à reprendre les dessins d'Éliphas Levi (Wirth 1889). Sur le retour aux dessins primitifs et sur la fixation de leur symbolisme kabbalistique, voir: Wirth 2014 [1927]: 43-48. 
21. «C'était une vierge orthodoxe à laquelle le Maître changeant quelques détails avait ajouté quelques ornements symboliques. Ce tableau passa plus tard chez le baron Spedalieri.») Note de l'auteur.

22. L'inventeur de cette machine, Józef Maria Hoene-Wroński (1778-1853), ancien officier d'artillerie des légions polonaises en France, entendait réconcilier la

mystique et les mathématiques et créa une nouvelle religion: le «séhélianisme». (Laurant1992: 61-63) Dans un autre contexte, on peut considérer le palais du Facteur Cheval comme une variante architecturale de ce principe encyclopédique d'écriture exposée.

23. La Bible de la liberté publiée en 1841 lui valut plusieurs mois de prison. Il poursuivit ensuite sa prédication à Évreux, puis adhéra à une société secrète de Lausanne, I'Ordre hermétique de la Rose-Croix universelle.

24. Adolphe Franck (1810-1893), figure méconnue de la philosophie spiritualiste, du libéralisme politique et du judaïsme français. II enseigne le droit au Collège de France

et introduit une valorisation

bienveillante de la kabbale,

qu'il considère comme «le cœur

et l'âme» du judaïsme: La Kabbale, ou la philosophie religieuse

des Hébreux (1843). [Rothschild et Grondeux 2012] de Montparnasse, a eu pour ambition de renouveler sous tous ses aspects l'affirmation visuelle de cette profession de foi en une réunification de la science et de la reliǵion. La mémoire encore vive des témoins, dans les dernières décennies du siècle, associe le maǵe à une collection de livres, d'objets-signes et de garants culturels qui semblent recomposer en style néomédiéval un moderne laboratoire-oratoire, avec ses tapisseries, ses objets rares, ses meubles sculptés. Manuscrits précieux et livres somptueusement reliés composent la vaste bibliothèque où veillent portraits et bustes de Rabelais, Voltaire, Rousseau. Vêtu d'une longue veste de velours, ornée de galons dorés, le mage reçoit ses visiteurs dans la chambre où il travaille et dort:

Éliphas Lévi s'était donné le plaisir d'édifier dans l'alcôve une couchette en bois d'acajou, avec baldaquin et colonne torses. Le lit était couvert d'une splendide courtepointe de velours pourpre brodée et surbrodée d'or: de la même étoffe, frangée d'or, étaient les rideaux et devant cette couche magnifique, sur laquelle reposait un moelleux coussin pareillement pourpre et or, était une marche également recouverte de velours. On retrouvait la même étoffe dans le cabinet de travail. Près de la fenêtre, un large fauteuil de bois sculpté, aux coussins brodés d'or, et une grande table de chêne, qui portait des papiers rangés avec ordre. Derrière le fauteuil, sur une petite table, le Prognomètre de Wronski; en face, contre le mur un tableau: Sainte Sophia 21. C'était là qu'Éliphas Lévi vivait et recevait.

(Chacornac 1989: 227-228)

Retrouvé chez un brocanteur versaillais, l'éniǵmatique proǵnomètre ou «machine à prédire » désigne Lévi comme le nouveau prophète de ce messianisme échafaudé par un philosophe et mathématicien polonais, et donné à voir à travers une figure hiéroǵlyphique en trois dimensions. Le biographe reprend, mot pour mot, la lecture de cette écriture en images que le mage en a donnée dans sa correspondance:

En voici à peu près la forme. Sa figure est celle de la lettre Schin. La double branche qui part du pied de la machine, se termine par deux boules de cuivre surmontées de deux pyramides triangulaires; l'une est le savoir divin, l'autre le savoir humain, partant de la même base et fonctionnant ensemble mais toujours opposés l'un à l'autre afin que l'harmonie résulte de l'analogie des contraires.

L'homme peut faire le tour de la sphère des sciences; jamais il ne rencontrera Dieu qui semble fuir devant ses recherches et qui est toujours caché par le ǵlobe c'est-àdire par l'épaisseur des choses. Cependant Dieu le polarise et lui fait équilibre même dans ses plus grandes erreurs.
Le globe qui symbolise la divinité se démonte et on y lit: tout ce qui doit être a été, est et sera. Autour du globe sont fixées quatre lettres découpées et mobiles A.B.X.Z, équivalent de Hé, Vav, Hé, Iod en signes algébriques, et qui ont la valeur de Tav, Schin, Beth, Aleph. De ce globe sortent deux branches articulées et munies de petits compas donnant la proportion de ce qui est en haut avec ce qui est en bas et du ǵlobe qui tourne avec son Zodiaque immobile.

Le globe du savoir humain porte une pyramide sur la pointe de laquelle le signe de Salomon se présente de tous côtés et la flèche tournée vers le globe est terminée par le pentagramme, signe de l'initiative et de l'autonomie humaine. [...]

Cette machine philosophique est toute une encyclopédie et le globe intérieur est chargé de longues équations que les plus forts mathématiciens de l'Académie des Sciences auraient sans doute de la peine à déchiffrer.

Sur la roue, qui dans les sphères célestes ordinaires porte les signes du Zodiaque, sont établies des portes qui s'ouvrent et se referment à volonté. Sur les portes sont écrits les noms des Sciences; sous les portes sont écrits à la main les axiomes fondamentaux de chacune d'elles. Il y a trente-deux portes et sur chaque porte le nom de trois sciences. Les axiomes sont d'une grande précision et tracés d'une manière très nette mais d'une écriture si fine qu'avec une loupe je lis encore assez difficilement.

(ibid. 137-139) 22

En l'absence de toute continuité d'une transmission orale - le «chuchotement des traditions» selon la belle expression d'Agrippa -, Éliphas Lévi affirmait avoir retrouvé par lui-même la science universelle que l'on croyait à jamais perdue, «ensevelie sous plusieurs cataclysmes successifs ». À vrai dire, c'est d'abord dans la riche bibliothèque de l'abbaye de Solesmes que celui qui, en 1838, se nommait encore AlphonseLouis Constant découvrit ces traditions textuelles alors qu'il songeait à nouveau à la prêtrise après une première tentative avortée ${ }^{23}$. Professant un socialisme mystique qui identifiait le royaume de Dieu à la société socialiste à venir, l'ami de Flora Tristan entendait réanimer, en autodidacte, le mouvement de la kabbale chrétienne en s'inspirant de l'étude scientifique qu'en proposait Adolphe Franck au milieu du XIX ${ }^{\mathrm{e}}$ siècle ${ }^{24}$.

\section{RÉINVENTER LE VERBE HIÉROGLYPHIQUE}

Au cœur de cette entreprise de vulgarisation, Lévi inscrit, comme il se doit, la question des noms divins. Selon la «Loi orale et secrète» donnée par Dieu 


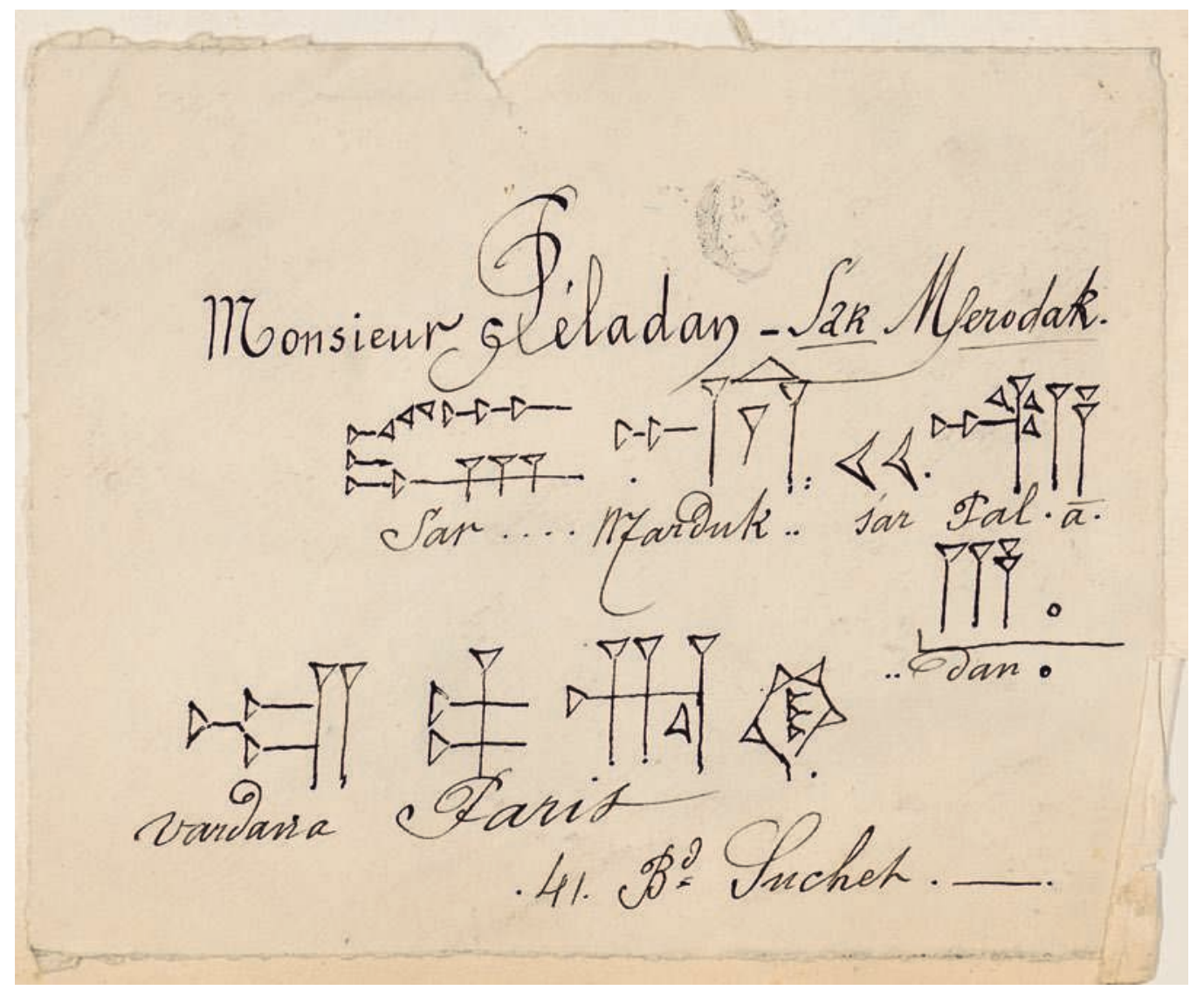

Enveloppe d'une lettre adressée à Joséphin Péladan, vers 1901-1910.

Bibliothèque nationale de France. Bibliothèque de I'Arsenal. Ms-13412, folio 16.

à Moïse en même temps que la «Loi écrite et publique» (la Torah), le tétraǵramme «YHVH», Yahvéh, a suscité de nombreuses spéculations. C'est au philosophe et théologien allemand Jean Reuchlin, rappelle le mage, que l'on doit sa conversion en un signe chrétien en plaçant la lettre hébraïque shin au centre, pour produire le pentaǵramme "YHSVH» (Yehoshua ou Jésus) qui fait du Christ la figure de l'Incarnation, en rendant audible le Nom ineffable composé uniquement de consonnes. La non-vocalisation de l'alphabet hébraïque demeure, en effet, comprise comme une forme d'occultation. De la tradition identifiée comme «kabbale des noms », il retient que les vingt-deux lettres hébraïques et les dix séphiroth ou émanations de la puissance divine constituent le matériau de la création du monde. Les procédés de mise en équivalence et de substitution des lettres, des symboles et des chiffres fascinent l'ancien prêtre qui pratique assidûment ce classique de l'écriture en langage chiffré, la Polygraphie et universelle escriture cabalistique de l'abbé bénédictin allemand Jean Trithème (1462-1516), lequel mettait la science des lettres au service de l'invocation des anǵes et protégeait ses recherches par des méthodes de chiffrement. Éliphas Lévi en retient que chaque lettre représente un mot, lui-même déterminé par un chiffre et qu'à partir de la numérotation des alphabets, on peut prendre pour clé le nombre d'un alphabet quelconque pour déterminer le sens d'une écriture occulte. On peut, aussi, traduire en lettres et en chiffres les signes astronomiques, les symboles chimiques, les écritures cunéiformes, les symboles maçonniques. À un confrère maǵnétiseur, Lévi détaille ses instruments:
«1) un alphabet polyǵlotte et comparé de toutes les langues anciennes; 2) la Clavicule de Salomon suivant Trithème; 3 ) le Protée ou la roue des Rose-Croix [le jeu de Tarot], qui est une synthèse hiéroǵlyphique de la langue universelle réalisée autrefois par l'alphabet de la Kabbale.» La conversion des signes et des figures peut dès lors mimer sur le plan spéculatif les principes adoptés à la Renaissance pour «arcaniser» les textes (Idel 1998):

L'alphabet comparé des langues anciennes et primitives les ramène toutes à une même source et conduit à l'intelligence des signes hiératiques et des symboles religieux. Chaque lettre est une synthèse hiéroglyphique dont les variétés mêmes, conformes aux génies des différents peuples, ne font que mieux ressortir l'unité. Dans ces alphabets l'idée du nombre est exprimée par le signe qui caractérise la voix, et à l'idée du nombre se joint inséparablement celle de l'être, du mouvement et de la vie. Aussi les éléments en sont-ils simples: c'est d'abord le point, puis la ligne droite, signes que nous avons conservés dans notre lettre $i$, correspondant au jod hébreux qui représente l'unité par excellence et doit être regardé comme le signe primordial du verbe. Le Jod est en effet la lettre mère de tout l'alphabet hébrä̈que, c'est la première lettre du nom de Jéhova et le signe 

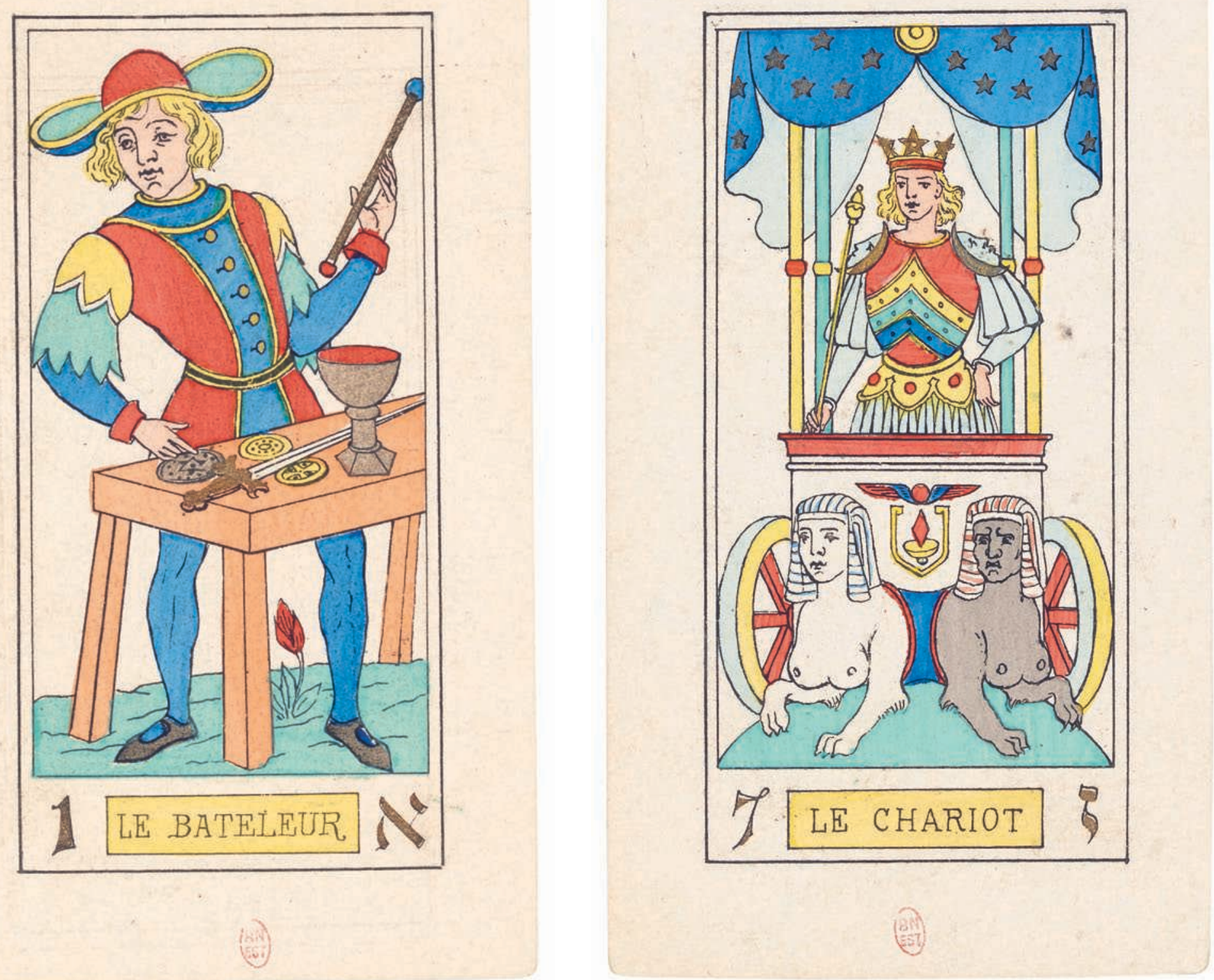

Jeu de tarot kabbalistique dit «des imagiers du Moyen Âge» d'Oswald Wirth, 1889. Chaque arcane correspond à une lettre de l'alphabet hébreu qui figure dans le cartouche inférieur. 22 cartes à jouer, lithographie coloriée au pochoir. Le Bateleur, Le Chariot.

Bibliothèque nationale de France, département Estampes et photographie, RESERVE KH-34 $(5,53)$-BOÎTE ECU. 

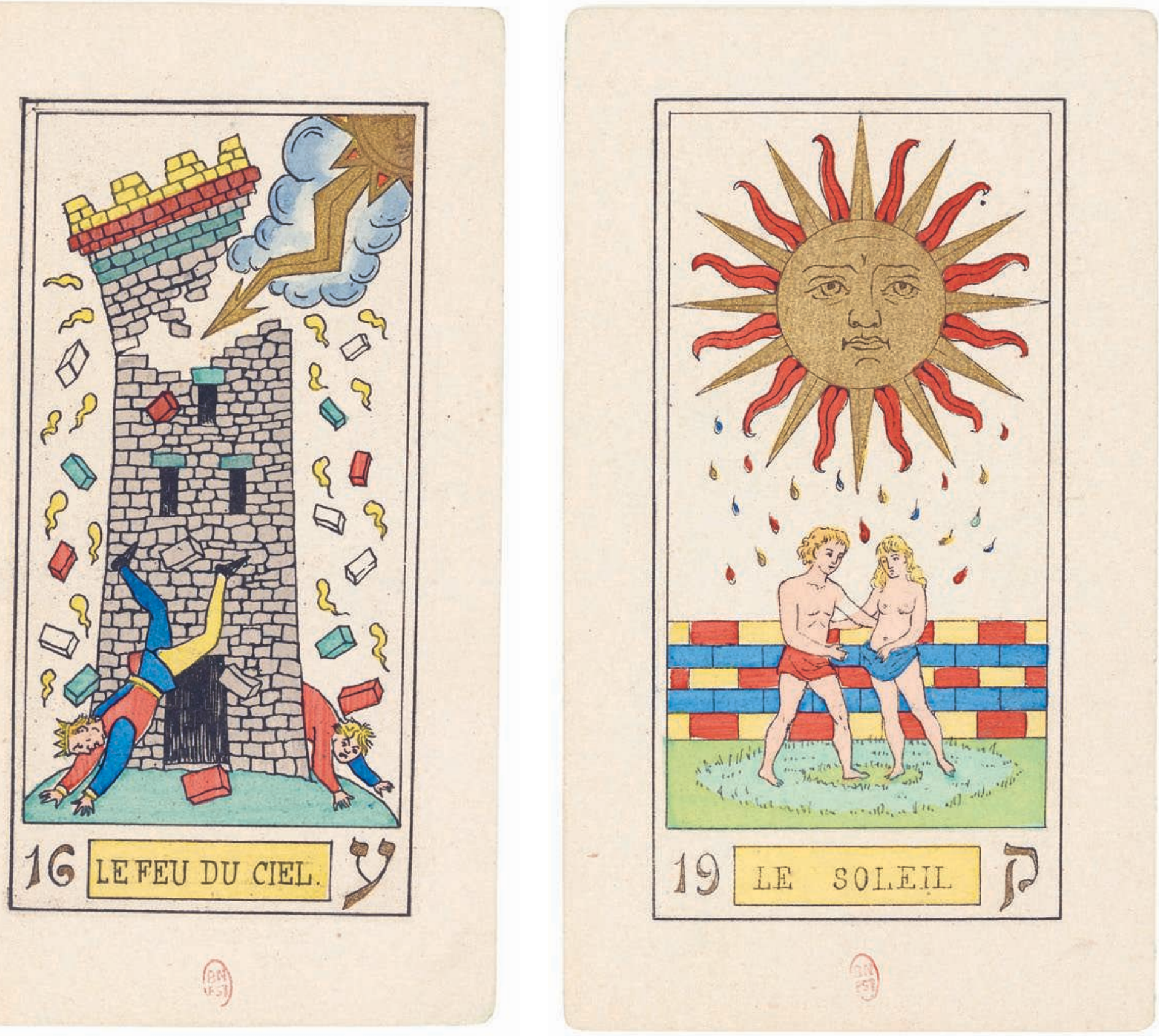

Jeu de tarot kabbalistique dit «des imagiers du Moyen Âge» d'Oswald Wirth, 1889. Chaque arcane correspond à une lettre de l'alphabet hébreu qui figure dans le cartouche inférieur. 22 cartes à jouer, lithographie coloriée au pochoir. Le Feu du ciel, Le Soleil.

Bibliothèque nationale de France, département Estampes et photographie, RESERVE KH-34 (5, 53)-BOÎTE ECU. 
25. L'importante collection de manuscrits formée au xVIII ${ }^{\mathrm{e}}$ siècle par Antoine-René de Voyer d'Argenson, marquis de Paulmy, est conservée à la bibliothèque de I'Arsenal à la bibliotr

26. Cette thèse est développée dans le livre VIII d'une somme inachevée, consacrée au Monde primitif analysé et comparé avec le monde moderne, dont neuf volumes paraissent entre 1775 et 1784 . Seul reste des superbes bibliothèques égyptiennes, celivre invisibl euptiennes, celi auxyeux des savants européens «... est en un mot le JEU DES TAROTS, jeu inconnu, il est vrai, à Paris, mais très connu en Italie, en Allemagne, même en Provence, \& aussibisarre parles figures qu'offre chacu par les figures qu'offre chacune de ses cartes, que par leur multitude» (Gébelin 1781: 365) Il enferme «l'Univers entier \& les États divers dont la vie de l'Homme est susceptible». Sur l'ambition du livre unique totalisant l'ensemble des connaissances, voir: Mercier-Faivre 1992. Sur I'histoire du tarot et une nouvelle lecture ésotérique, voir: Van Rijnberk 2019. générateur de tous les signes cabalistiques. Vient ensuite la ligne courbe, puis les combinaisons des diverses lignes; et aucune de ces combinaisons n'est faite au hasard: la ligne courbe fut empruntée au mouvement apparent et les assemblées des lignes exprimèrent les combinaisons soit de nombre soit d'idées exprimées par chaque caractère. Vous comprenez déjà que, cette observation une fois faite, il est facile de trouver une lettre dans un signe quelconque, de trouver à cette lettre une place dans un des alphabets primitifs et de lui assigner une signification, soit numérale, soit phonétique, soit philosophique, absolue

(Chacornac 1989 [1926] : 156-157)

Mais s'inscrire dans la prestiǵieuse liǵnée des maǵes exiǵe non pas tant d'apprendre à manipuler un héritage linguistique que de recréer pour soi la lanǵue qui permet de s'installer en position d'interlocuteur de l'invisible. Éliphas Lévi compose son propre livre d'incantations en créant un répertoire personnel de trente-six talismans auquel il donne le vénérable nom de Clavicules de Salomon, soit le manuel de magie rituelle le plus répandu à l'époque moderne.

Probablement d'origine grecque, une traduction latine manuscrite a introduit ce traité dans le monde latin au milieu du XIII ${ }^{\mathrm{e}}$ siècle, avant que ne paraisse en 1629 la première édition imprimée à Rome qui relance les copies, puisque seule une appropriation personnelle permet au magicien de détourner les sacrements pour entrer en rapport avec les démons, à l'abri des lettres sacrées et des noms divins qui le protègent d'une mort certaine (Davies 2009) ${ }^{25}$. Or, à la différence des livrets populaires qui, sous ce même nom, diffusent les noms et les figures d'une démonologie chrétienne, c'est l'abondante angéoloǵie hébraïque que convoque Lévi sous le double aspect d'une liste de noms et d'éniǵmatiques figures associés, deux par deux, à un registre de bienfait ou de puissance. Lettres hébraïques, grecques et latines, figures géométriques, nombres et emblèmes - baǵuette, chandeliers, cristal, torche, poignard - composent les idéoǵrammes de cette lanǵue personnelle que les disciples du mage révéleront à un plus large public, lonǵtemps après sa mort (Lévi 1895).

Cette inventivité graphique pose deux questions comment comprendre la valorisation des lettres hébraïques? Comment lire les figures que Lévi désigne comme des emblèmes? Bien que la question des origines ait été définitivement écartée, en 1865-1866, par les statuts de la Société de linguistique, Lévi, comme le fera après lui Guaita, se réclame du mythe culturel de la langue hébraïque proposé par le linguiste et théosophe Fabre d'Olivet, en opposition à la thèse conventionnaliste des oriǵines du lanǵage.

Ce savant ne se proposait-il pas de restituer un hébreu authentique et perdu pour détacher la Bible de son interprétation chrétienne et retrouver l'origine de «la Parole» primitive décelable sous la diversité des langues historiques (Fabre d'Olivet 1815) ? À cette quête avait succédé une entreprise analogue de restauration de la langue occitane, ce qui autorise la critique contemporaine à reconnaître, dans ce geste répété d'exploration de la langue comme scène des oriǵines, une théorie de la langue mère à restaurer dans sa force primitive, à travers une remontée dans le temps pour se retrouver aux oriǵines de «la Parole». Faire jaillir de chaque mot les trois sens littéral, figuré, hiéroǵlyphique: tel apparaît, alors, le mérite de Fabre d'Olivet pour un praticien comme Éliphas Lévi, tandis que Guaita, davantage porté aux spéculations sémiotiques, définit la spécificité du mode hiéroǵlyphique comme suppléant à l' «l'impuissance du langage phonétique » (Guaita 1890: 177).

S'aǵissant des emblèmes, ils appartiennent à un corpus d'imaǵes - les cartes du jeu de tarot dont Éliphas Lévi dénonce la réduction contemporaine à un matériel divinatoire, au nom du légendaire élaboré, au XVIII ${ }^{\mathrm{e}}$ siècle, par le protestant et franc-maçon Antoine Court de Gébelin. Cet érudit assimilait les cartes du tarot aux feuilles détachées du livre le plus ancien du monde, enfermant toute la sagesse éǵyptienne dictée par le dieu Thot en personne ${ }^{\mathbf{2 6}}$. Lévi met le mythe en pratique: les vingt-deux lames ou arcanes majeurs du jeu doivent être mises en correspondance avec les vingt-deux lettres de l'alphabet hébraïque. Ainsi, le Bateleur, première lame, doit être associé à aleph, première lettre, et cette lecture en clé kabbalistique sera systématiquement reprise par la génération suivante d'occultistes, tout en rejetant l'origine éǵyptienne du jeu. Mais quelles exigences motivent le recours à un répertoire d'images dès lors sans cesse repris et recréé par les générations successives d'opérateurs? Pour apporter un début de réponse à cette question il faudrait sortir de l'argumentation idéologique afin d'observer une pratique concrète.

Heureusement, un remarquable document se prête à cette exiǵence. Il s'agiit des leçons qu'Éliphas Lévi adressait sous forme de lettres hebdomadaires au baron sicilien Nicolas-Joseph Spedalieri qui fut, parmi ses douze disciples, son élève et ami le plus notoire. Du millier de lettres échangées entre 1861 et 1874 , une partie est aujourd'hui publiée comme un «cours de philosophie occulte» qui déplie l'équivalence «Tarot, rota, Torah» condensée dans le monogramme que le mage s'est donné pour emblème (Lévi 1988).

Ouvrons-le.

L'enseiǵnement commence, en novembre 1861, avec l'envoi à l'élève d'une copie de ces "Saintes Clavicules» dont le maǵe souliǵne, d'emblée, la correspondance avec les figures du jeu: les figures «représentent des bâtons, des coupes, des épées et des cercles comme les hiéroǵlyphes du Tarot» (ibid.: 2). Pour comprendre les lettres et les nombres de ces talismans, précise l'auteur, il faut un tarot italien ancien, et de promettre à l'élève de lui en apprendre l'usage dès qu'il pourra en disposer. Mais, même à Marseille, il est difficile de se procurer l'objet. «Je vais, assure le maître, m'occuper de vous arranger un tarot et je vous l'enverrai. » Le 30 novembre, il peut confirmer l'envoi d'un tarot italien «corrigé et rectifié». La suite 

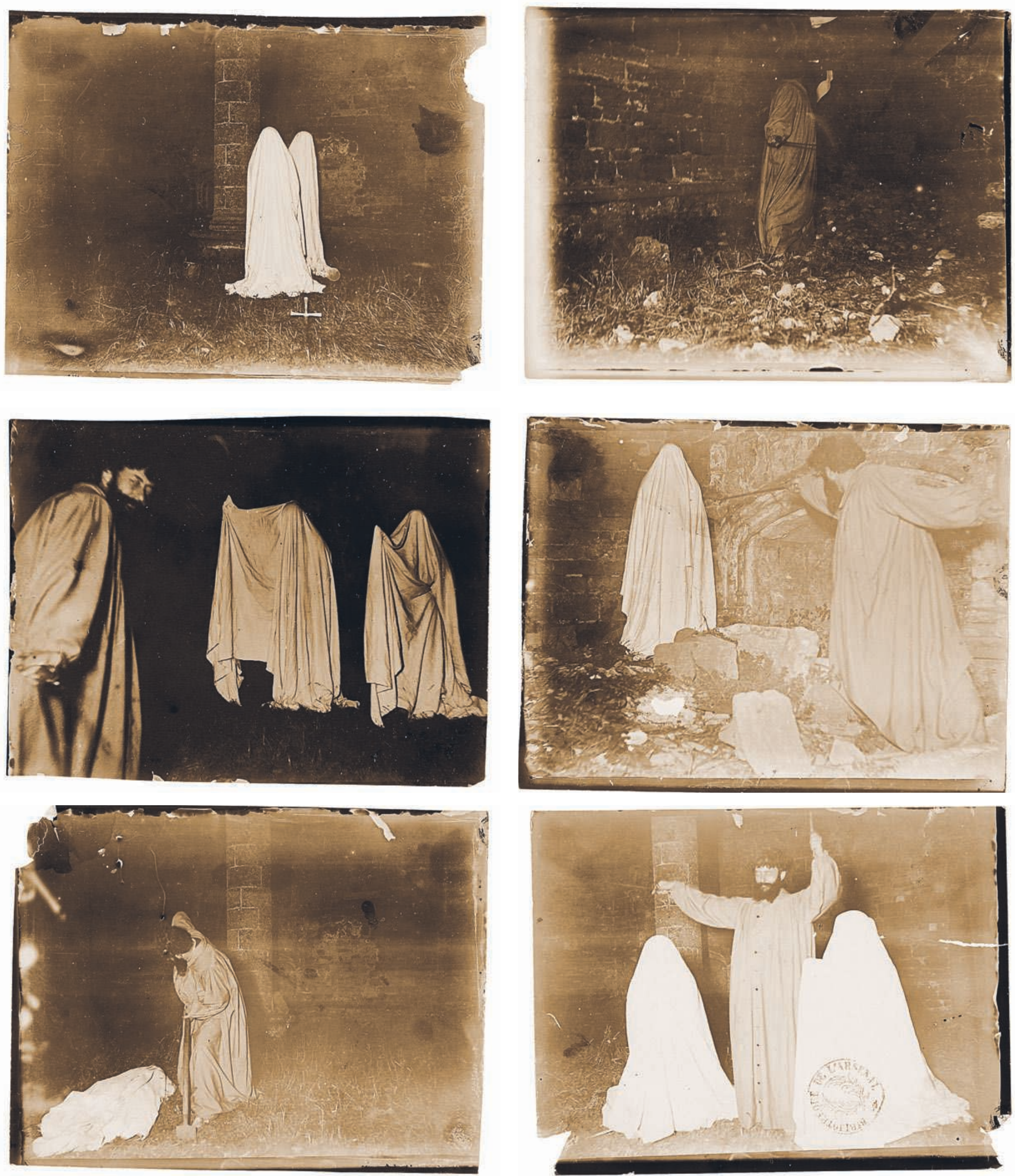

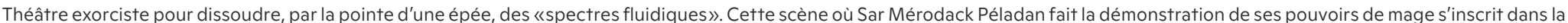
dissidence d'une Rose-Croix catholique qui provoque, en 1890, la rupture avec Guaita. Bibliothèque nationale de France. Bibliothèque de I'Arsenal. Ms-13412. 
27. Le Séfer Yetsira ou «Livre de la Création», est un traité de cosmogonie juive rédigé entre le me etle vie siècle. Attribué à Abraham, il relate

la création du monde au moyen des vingt-deux lettresde l'alphabet hébraïque.

Il est traduit de l'hébreu

par Henri et Hector Durville en 1913. de la correspondance expose le principe qui a commandé le choix des noms et des figures pour la composition des talismans. Le tarot est la clé des lettres et des nombres; ceux-ci sont la clé du tarot; les quatre lettres du tétraǵramme divin correspondent au bâton, à la coupe, à l'épée et au denier du jeu; le Sepher Jezirah est la référence textuelle des talismans, des lettres, des nombres, du tarot ${ }^{27}$. Puis, chacune des lettres hébraïques est commentée à travers sa mise en correspondance avec l'un ou l'autre arcane qui en fournit une figuration humaine: on peut, ainsi, reconnaître la lettre aleph dans la figure du Bateleur. Ce faisant, l'élève apprend à lire une lettre comme une image, et une image comme une lettre; il apprend à jouer avec les lettres aussi bien qu'à convertir l'écriture en dessin et le dessin en écriture; il apprend, en somme, la tradition médiévale de l'écriture par figure.

Quant à l'utilité sociale de cette Haute Maǵie, Éliphas Lévi l'évoque succinctement, nous laissant seulement entrevoir de possibles usages: divinatoires, lorsque au consultant qui fournit des noms et des chiffres Trithème «rendra trois, cinq ou sept pages de traduction qui seront de véritables oracles...»; de divertissement ou, à l'inverse, de «moralisation» d'innovations religiieuses concurrentes, comme les modernes pratiques d'évocation des esprits. Il faut, encore, se tourner vers les souvenirs de son éditeur pour apercevoir le contraste entre les étourdissantes leçons que le mage de Montparnasse donnait à ses élèves et les techniques du thérapeute au travail. À l'érudition fait place l'éclectisme de la modernité magnnétique, voire les usages les plus communs de la coutume chrétienne: pour un ouvrier qui fait des «évocations », une prière à lire chaque soir, au moment du coucher, écartera «les esprits des ténèbres » en faisant apparaître le mage dans une vive lumière (Chacornac 1989 [1926] : 177).

\section{LES JUSTICES TRANSCENDANTES}

C'est à son disciple Oswald Wirth que Stanislas de Guaita déléguera le soin de composer de nouvelles figures pour faire parler les lettres hébraïques, tandis que lui-même assume en écrivain les aléas de la fonction de mage au sein d'un collège dont il a projeté la création dès sa rencontre avec Joséphin Péladan. Le compagnonnage imaginaire a, très vite, fait place au partage au quotidien de l'excentricité d'une vie d'artiste en régime ésotériste. Les deux amis rêvaient d'un collectif de «frères», à la fois centre de recherches expérimentales et société hiérarchisée ordonnée par une pratique rituelle. L'Ordre kabbalistique de la Rose-Croix a vu le jour, en 1888, immédiatement après une opération de «justice transcendante» qui ne sera rendue publique que quelques années plus tard.

L'affaire éclate dans les journaux au lendemain de la mort d'un certain Joseph-Antoine Boullan en janvier 1893. Ce prêtre a quitté l'Église en 1875 pour devenir le «Pontife Suprême» d'un «Carmel Trématique Eliaque », alors qu'il venait d'être condamné pour exercice illégal de la médecine. Dans son édition du 7 janvier, le journal L'Éclair annonce la mort d'un officiant de «messes noires», familier de «vieux grimoires». Accusation discutable, admet le rédacteur anonyme, puisque le prêtre a pu, pour un temps, gaǵner la confiance de personnes cultivées, tel l'écrivain Huysmans qui a fini par en dévoiler la vraie nature dans Là-Bas (1891), sous les traits du chanoine Docre (Bricaud 1927 : 82). Le 9 janvier, c'est au tour du journaliste Jules Bois d'avancer un autre diaǵnostic dans les colonnes de Gil Blas : l'abbé Boullan est mort victime d'un envoûtement dont il accusait formellement Stanislas de Guaita, Oswald Wirth et Péladan, les animateurs de la société rosicrucienne. À l'appui

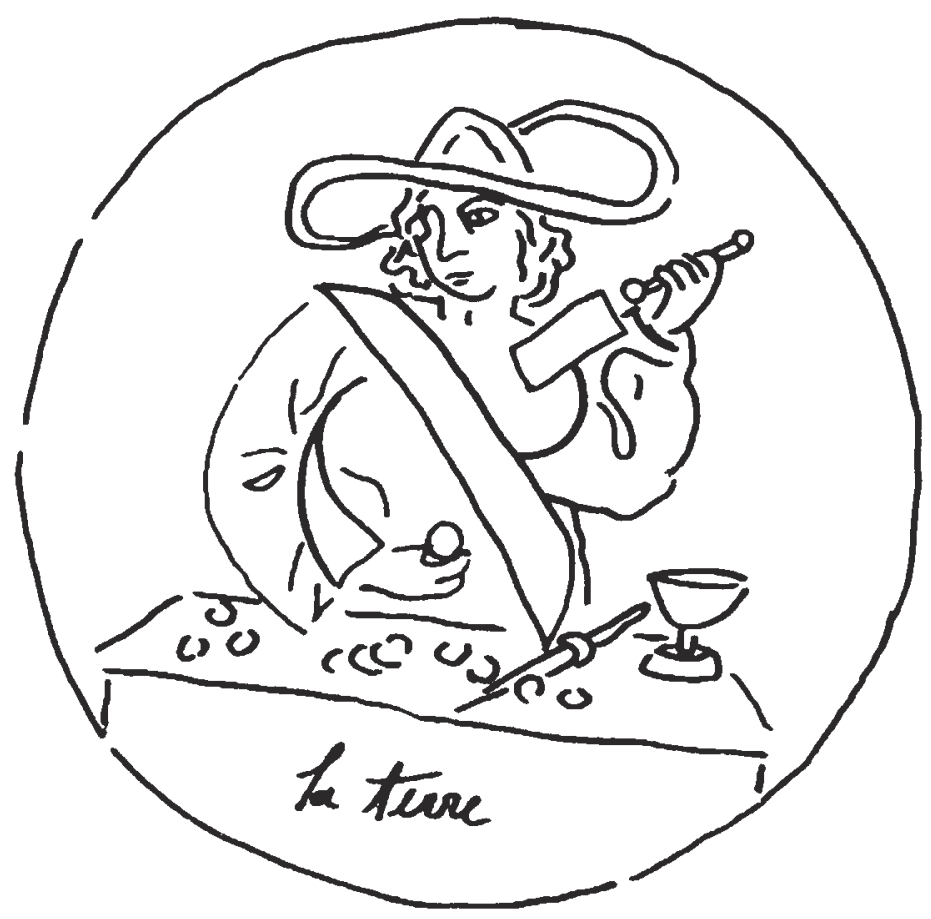


de ce verdict, le journaliste détaille les pressentiments de la victime, les visions prophétiques de deux adeptes, la réputation de sombre maǵicien du principal coupable - Guaita -, ainsi que les acquis de la science médicale qui, avec Charcot, Luys, de Rochas, reconnaît «la puissance des envoûtements». Le 10 janvier, Huysmans reconduit ces accusations dans un entretien accordé au Figaro: «Il est indiscutable que de Guaita et Péladan pratiquent quotidiennement la magie noire. Ce pauvre Boullan était en lutte perpétuelle avec les esprits méchants qu'ils n'ont cessé, pendant deux ans, de lui envoyer de Paris. » Dans les jours suivants, le journaliste riposte longuement à une note de Guaita parue dans Le Figaro, en mêlant deux registres explicatifs, celui, psychologique, de la haine inexorable et celui, magique, de l'«envoûtement collectif» visant une victime solitaire. Pour toute réponse, l'accusé tourne en ridicule les pouvoirs maléfiques qui lui sont imputés et contreattaque: il donnera ses explications «sur le pré » à ses calomniateurs. Huysmans esquive l'affrontement en revenant sur ses propos, Jules Bois relève le défi. On échangea deux balles à la tour de Villebon, à Meudon. Il n'y eut pas de victime et la presse tourna la page.

À vrai dire, seuls les lecteurs de deux ouvrages également parus en 1891 pouvaient comprendre ces invectives et ces accusations réciproques. L'un a sombré dans l'oubli, l'autre est entré dans notre histoire littéraire. Dans Le Temple de Satan, second tome de ses Essais de sciences maudites, Stanislas de Guaita procède au «baptême de lumière», en rendant publique la sentence que le tribunal d'honneur qui s'est réuni quatre ans plus tôt a prononcée le 23 mai 1887 à l'encontre de Joseph-Antoine Boullan. Dans Là-Bas, Huysmans abandonne le roman naturaliste pour une enquête sur les formes anomiques de mysticisme parmi lesquelles figure en bonne place, sous le nom de «Dr Johannès», le pontife du carmel lyonnais. Ainsi celui qui s'employait à dénoncer les démonologues du passé vient, à son tour, en occuper la place pour combattre un «faux» mage en mimant les formes judiciaires de l'enquête: il rassemble des documents, il établit des preuves; mais, assimilée à un rite religieux - le «baptême de lumière»- la publication du juǵement délègue à la science la condamnation du coupable. Il devient à son tour victime de cet imaginaire sorcellaire transmis par la presse et par le roman moderne qui, lui-même, intègre les techniques de l'enquête sociale pour inventer une autre forme d'écriture ${ }^{\mathbf{2 8}}$.

$*$

En régiime occulte, version fin de siècle, le principe fondamental de la fraternité entre les hommes implique non une dissolution, mais une réactivation du lien religieux inscrite dans une forme paradoxale de primitivisme historique: l'imaginaire des origines qui accompaǵne, plus larǵement, une crise reliǵieuse, se nourrit d'une fascination pour toutes ces techniques intellectuelles très sophistiquées qui, dans le passé des sociétés occidentales, ont accompagné la confrontation à d'autres traditions culturelles et favorisé l'invention de nouvelles langues graphiques. Parler ou écrire, écrire ou dessiner? En cette fin du XIX siècle, les «sciences maudites» entendent subvertir ces alternatives en suppléant à l'appauvrissement des langues et des textes imprimés, qu'elles s'emploient à faire parler en les ressaisissant par l'image. Comme toute invocation de l'originaire, celle-ci fait le pari de la modernité en participant à ce retour de l'image dans l'écrit qui sous-tendra le développement d'un art de la typographie où l'illustration, les affiches, les jeux avec la lettre vont redécouvrir le pouvoir de sémantisation des signes graphiques. Mais suffit-il de rompre avec les codes institués pour que, des signes, puissent de nouveau surǵir de réelles présences? Entre les mains du maǵe cette pensée figurative, qui affirme une filiation entre l'écrit et l'image, se veut au service d'une théorie de la parole créatrice en un temps où les religions du Livre s'éprouvent comme orphelines d'une parole perdue. Or les innombrables textes qui se sont employés à relayer cette utopie sont devenus illisibles, voués qu'ils étaient à se répéter indéfiniment pour réitérer la situation d'énonciation propre à l'oralité perdue.

Pourtant, les circulations culturelles qui traversent aussi bien les textes que leurs mises en pratique rappellent obstinément une exiǵence de sens, en même temps qu'elles favorisent un changement de statut de la matière manipulée, qui installera proǵressivement les «sciences maudites» dans la modernité littéraire. André Breton, on le sait, avait une affection particulière pour Éliphas Lévi ${ }^{29}$; un peu plus tard, ce sont les poètes rassemblés autour de la revue La Tour de feu qui recréent un «tarot de Jarnac» pour poursuivre l'inspiration surréaliste d'une poésie prenant le relai de la maǵie ${ }^{\mathbf{3 0}}$; mais demandons, plutôt, à Italo Calvino d'éclairer ce déplacement esthétique. Dans Le Château des destins croisés, des chevaliers se retrouvent à une table d'hôtes et au moment de conter leur aventure, la parole leur fait défaut. Ils ont alors recours aux miniatures raffinées du tarot Visconti qui permet à chacun d'eux d' "écrire par figure» son histoire. La Taverne des destins croisés, la nouvelle qui suit la précédente, engendre de la même façon une série de récits à partir du tarot de Marseille, dont les gravures plus ǵrossières appellent une autre langue, où s'entrecroisent les histoires et les images. Sans doute vaut-il mieux renoncer à la quête d'une lanǵue mère pour qu'advienne le sens qui circule entre les signes, pour que naissent les histoires qui libèrent «le rêve que la parole porte en soi - nous dit Italo Calvino - et qui, passant par celui qui écrit, se libère en le libérant ${ }^{31}{ }$.
28. Miné par la consommation de cocaïne et de morphine, Guaita meurt le 17 décembre 1897, à 38 ans. Huysmans, converti au christianisme, reconnaîtra, en privé, le satanisme de Boullan.

29. Nicolas Flamel inspire simultanément André Breton dans le Second Manifeste du surréalisme et Robert Desnos dans «Le mystère d'Abraham Juif», pour la revue Documents, $n^{\circ} 5$, octobre 1929. Breton affirme une «analogie de but» entre les recherches alchimique et surréaliste; Abraham Juif et surrealiste; Abraham Juif
et Hermès sont des initiateurs comparables à Rimbaud et à Lautréamont, si l'on voit dans la pierre philosophale la métaphore du pouvoir de l'imaginaire qui substitue l'exigence poétique à la quête métaphysique.

30. «Le nouveau tarot de Jarnac», La Tour de feu, mars 1974, cahier 121. Cette revue de création poétique est fondée en 1933 par le «poètetonnelier» Pierre Boujut, socialiste libertaire.

31. Calvino 1976: 112. II s'agit pour l'écrivain d'assimiler les figures du tarot à une machine à engendrer des récits. 


\section{Bibliographie}

Barrès, Maurice

1898 Stanislas de Guaita: un rénovateur de l'occultisme. Souvenirs. Paris, Chamuel.

\section{Beaufils, Christophe}

1993 Joséphin Péladan

(1858-1918): essai sur une

maladie du lyrisme. Grenoble, Jérôme Millon.

\section{Béhar, Pierre}

1996 Les Langues occultes de la Renaissance: essai sur la crise intellectuelle de l'Europe au XVI $I^{e}$ siècle.

Paris, Desjonquères.

\section{Bénichou, Paul}

1988 Les Mages romantiques. Paris, Gallimard.

Bois, Jules

1895 Le Satanisme et la magie Paris, Léon Chailley.

\section{Boudet, Jean-Patrice}

2006 Entre science et nigromance. Astrologie, divination et magie dans l'Occident médiéval (XII'-XV siècle). Paris, Editions de la Sorbonne.

\section{Bricaud, Joanny}

1927 L'Abbé Boullan (Docteur Johannès de "Là-Bas») sa vie sa doctrine et ses pratiques magiques. Paris, Librairie générale des sciences occultes Chacornac frères.

\section{Calvino, Italo}

1976 Le Château des destins croisés. Paris, Seuil.

\section{Chacornac, Paul.}

1989 [1926] Éliphas Lévi (1810-1875), rénovateur de l'occultisme en France. Paris, Editions traditionnelles.

Christin, Anne-Marie

1995 L'Image écrite ou la déraison graphique. Paris,

Flammarion.

\section{Court de Gébelin, Antoine}

1781 Monde primitif analysé et comparé avec le monde moderne considéré dans son génie allégorique et dans les allégories auxquelles conduisit ce génie, t. VIII. Paris, Boudet [etc.]

\section{Coxe, Frédérick}

2014 «La bibliothèque occulte de Stanislas De Guaita»,

bloǵ Bibliophilie.com, 5, 7, 9, 10 janvier.

\section{Davies, Owen.}

2009 Grimoires: A History of Magic Books. Oxford, Oxford University Press.

Desnos, Robert

1929 «Le mystère d'Abraham Juif », Documents 5 : 233-237.

\section{Desormeaux, Daniel}

2001 La Figure du bibliomane: histoire du livre et stratégie littéraire au XIX ${ }^{e}$ siècle.

Saint-Genouph, Nizet.

Dufay, Pierre,

1935 «L'Abbé Boullan et le "chanoine d'Ocre" », Mercure de France 882: 509-527.

\section{Edeline, Francis}

2009 «Les fonctions sémiotique et heuristique des symboles chimiques ou de l'icône au symbole et retour », Protée 37 (3) : 45-56.

\section{Eco, Umberto}

1989 Lo strano caso della Hanau 1609. Milan, Bompiani. 1997 [1994] La Recherche de la langue parfaite dans la culture européenne.

Paris, Seuil.

Fabre, Daniel.

1985 «Le livre et sa maǵie», in Roger Chartier (dir.) Pratiques de la lecture. Marseille, Rivages.

Fabre d'Olivet, Antoine 1815 La Langue hébrä̈que restituée et le véritable sens des mots hébreux rétabli et prouvé par leur analyse radicale. Paris, Barrois l'aîné Eberhart.

\section{Farout, Dominique}

2016 «De la Renaissance à la Restauration: quelques étapes du déchiffrement des hiéroglyphes », Les Cahiers de l'École du Louvre 9: 1-40.

\section{Festugière, André-Jean}

2014 La Révélation d'Hermès Trisméóiste, Concetta Luna, Henri Dominique Saffrey et Nicolas Roudet (éd.). Paris, Les Belles Lettres.

Flamel, Nicolas

2019 [1624] His Exposition of the Hieroglyphicall Figures, Laurinda Dixon (éd.). Oxford, Routledge revivals.

Forshaw, Peter

2006 "Alchemy in the Amphitheatre: Some Considerations of the Alchimical Content of the Engravings in Heinrich Khunrath's Amphitheatre of Eternal Wisdom (1609) », in Jacob Wamberg (dir.), Art and Alchemy. Copenhague, Museum Tusculanum Press : 195-220.

\section{Grévin Benoît et Véronèse,} Julien

2004 "Les "caractères" magiques au Moyen Âg (XII ${ }^{\mathrm{e}}$-XIV ${ }^{\mathrm{e}}$ siècle) », in Bibliothèque de l'École des chartes 162 (2) : 305-379.

Guaita, Stanislas (de) 1890 Essais de sciences maudites, t. I : Au seuil du mystère. Paris, Georges Carré. 1915 [1891] Le Serpent de la Genèse, t. I : Le temple de Satan. Paris, Durville.
Huysmans, Joris Karl. 2008 [1891] Là-Bas. Paris, Gallimard.

\section{Idel, Moshe}

1998 La Cabale: nouvelles perspectives. Paris, Cerf.

\section{Ingold, Tim}

2011, Une brève histoire des lignes, trad. de l'anǵlais par Sophie Renaut.

Bruxelles, Zones Sensibles.

Jollivet-Castelot, François

1998 «L'alchimiste»,

L'Initiation 1 (n. s.) : 9-19.

\section{Kahn, Didier}

2007 Alchimie et paracelsisme en France à la fin de la Renaissance (1567-1625). Genève, Droz.

\section{Laurant, Jean-Pierre}

1992 L'Ésotérisme chrétien en France au XIX siècle. Lausanne, L'Âge d'Homme.

\section{Lévi, Éliphas}

1861 La Clef des grands mystères: suivant Hénoch, Abraham, Hermès Trismégiste et Salomon. Paris, G. Baillière.

1895 Clefs majeures et clavicules de Salomon. Paris, Chamuel.

1930 [1856] Dogme et rituel de la haute magie. Paris, Chacornac Frères.

1988 Numérologie et kabbale: cours de philosophie occulte. Québec, De Mortaǵne.

\section{Mandosio, Jean-Marc}

2005 «La création verbale dans l'alchimie latine du Moyen Âge », ALMA (Archivium Latinitatis Medii Aevi) $63: 137-147$

\section{Ménard, Louis}

1866 Hermès Trismégiste. Paris, Didier.

Mercier-Faivre, Anne-Marie

1992 «Le Monde primitif d'Antoine Court de Gébelin ou le rêve d'une encyclopédie solitaire », Dix-Huitième siècle 24 : 353-366.

\section{Obrist, Barbara}

2003 «Visualization in Medieval Alchemy», HYLE International Journal for Philosophy of Chemistry 9 (2) 131-170.

\section{Papus}

1888 Traité élémentaire de science occulte. Paris, Carré.

\section{Péladan, Joséphin} 1884 La Décadence latine (éthopée), t. I : Le Vice suprême. Jules Barbey d'Aurevilly (préf .), Félicien Rops (frontispice).

Paris, Chamuel.

\section{Philipon, René}

1899 Stanislas de Guaita et sa bibliothèque occulte. Paris, Dorbon.

\section{Postel, Guillaume}

1899 Clef des choses cachées dans la constitution du monde de l'éternelle vérité. Paris, Bibliothèque Chacornac.

Principe, Lawrence $M$. et Newman, William R.

2001, «Some Problems With

the Historiography of

Alchemy », in William R.

Newman et Anthony Grafton,

Secrets of Nature: Astrology and Alchemy in Early Modern Europe. Cambridge, Mass. The MIT Press: 384-431.

\section{Rothschild, Jean-Pierre} et Grondeux, Jean (dir.) 2012 Adolphe Franck: philosophe juif, spiritualiste et libéral dans la France du XIX ${ }^{e}$ siècle. Actes du colloque tenu à l'Institut de France le 31 mai 2010. Paris, Brepols.

\section{Schlanger, Judith E.}

1967 «La lanǵue hébraïque, problème de linguistique spéculative », Revue internationale de philosophie 21 (82-4): 486-507.

\section{Simon, Fabien}

2018 «Atlas des lanǵues. Prendre la mesure linguistique du monde à l'époque moderne (portfolio)», Terrain 70 [en liǵne], disponible sur : http:// journals.openedition.org/ terrain/17434 (consulté le 7 octobre 2020).

\section{Van Rijnberk, Gérard}

2019 [1947] Le Tarot: histoire, 
Siladay-Sak Merodak.

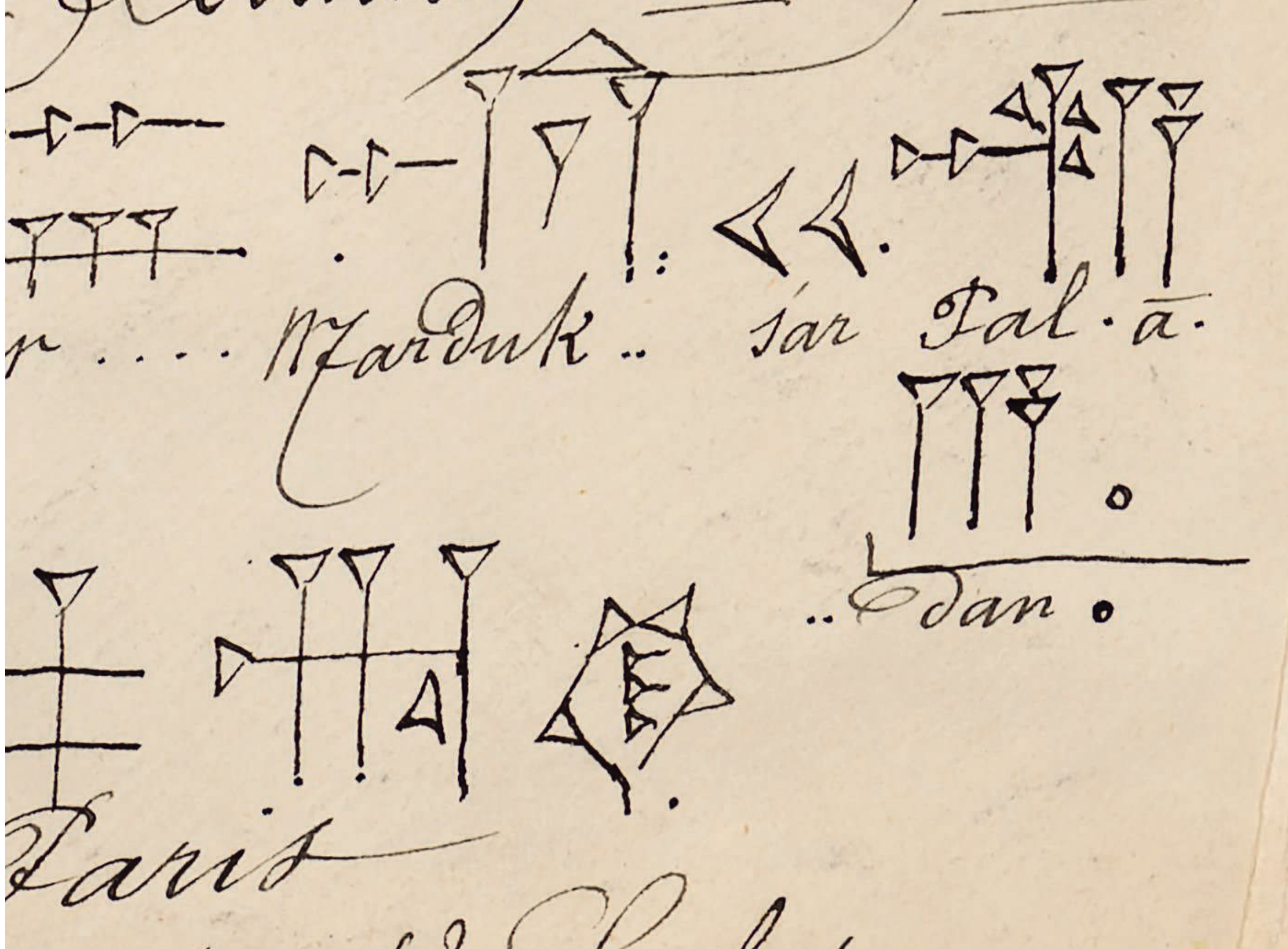

41. og: Suchet. 